\title{
Les ingénieurs chimistes en France entre les deux guerres Une analyse à partir d'une lecture de L'ingénieur chimiste (1919-1939)
}

\author{
André GRELON ${ }^{*}$
}

\section{Résumé}

L'étude du bulletin mensuel du Syndicat des ingénieurs chimistes français entre les deux guerres permet d'analyser les intérêts, les inquiétudes, les représentations sociales, les revendications de ce groupe professionnel. Né en 1919, ce syndicat est membre d'une Union des syndicats d'ingénieurs français (USIF), regroupant avec lui un syndicat d'électriciens et un syndicat de mécaniciens. Il rassemble des hommes très marqués par le vécu de la guerre qui vient de s'achever et qui s'estiment mal considérés sur le plan professionnel. Ils attribuent les causes de cette dépréciation au fait que les entreprises chimiques ne sont pas dirigées par des chimistes et qu'ils sont cantonnés le plus souvent à des emplois de contremaîtres. Ils dénoncent aussi les ingénieurs étrangers qui occupent les places des Français. Enfin, ils sont persuadés qu'il y a trop d'ingénieurs et que cela pèse sur les emplois et les salaires. Néanmoins, ils suivent avec attention la question des rémunérations et obtiennent des accords avec l'Union patronale. Des questions qui devraient les concerner beaucoup comme la rémunération des brevets ou les maladies professionnelles sont peu abordées. En revanche, le syndicat se mobilise sur la question des ingénieurs âgés dont il est persuadé qu'ils sont réduits au chômage après des années de loyaux services. C'est une thématique qui, à l'époque, touche l'ensemble du monde des ingénieurs. Toutefois, aucune étude statistique ne vient conforter cette hypothèse. Enfin, le syndicat mène une longue campagne pour obtenir de l'État la création d'un diplôme DPLG d'ingénieur des arts chimiques qui mettrait ses possesseurs au niveau de recrutement des anciens élèves des grandes écoles. Cependant, les négociations avec le ministère n’aboutiront jamais. En 1936, la période du Front populaire concerne aussi les ingénieurs. Pour pouvoir participer aux négociations sur les conventions collectives, le syndicat doit exclure de ses rangs les patrons qui en étaient membres. Les différents syndicats d'ingénieurs sont amenés, malgré leurs différences et leurs querelles, à se regrouper en une Fédération nationale des syndicats d'ingénieurs pour pouvoir peser au sein des partenaires sociaux.

\footnotetext{
*André GRELON, Directeur d'études à l'EHESS.
} 


\title{
Mots clés
}

Chômage des ingénieurs. Conventions collectives. Diplôme d'ingénieur chimiste. Diplôme d'État. Formation en chimie. Ingénieurs des poudres. Ingénieurs étrangers. Syndicat des ingénieurs chimistes français (SICF). Union des syndicats d'ingénieurs français (USIF). Union des industries chimiques (UIC).

\begin{abstract}
A study of the monthly bulletin of Union of French Chemical Engineers (Syndicat des ingénieurs chimistes français) during the interwar period allows an analysis of the interests, anxieties, social representations, and claims of this professional group. Founded in 1919, this union was a member of the Federation of Unions of French Engineers (Union des syndicats d'ingénieurs français, USIF), along with union of electrical and mechanical engineers. It brought together a group of men deeply affected by the recently ended war and who considered their profession did not enjoy the status it deserved. They attributed this lack of recognition to the fact that chemists, who were limited to lower management positions, did not manage chemical companies. They also denounced foreign engineers taking jobs from French ones. Lastly, they were convinced that there were too many engineers, which had a negative impact on jobs and salaries. Thus they were very attentive to the issue of pay and sought to negotiate agreements with the employers' Union.
\end{abstract}

Questions related to payments for patents or industrial diseases are rarely discussed despite their importance for the profession. Nevertheless, the union did mobilize around issues that concerned senior engineers, notably the prospect of unemployment after years of faithful service. Meantime no statistical studies reinforce such a hypotheses. Finally, the union supported a long campaign for the State to create a Chemical Arts Engineer diploma, with the idea that certified engineers could then be recruited at the same level as those graduating from the elite institutions of the Grandes Écoles. But the negotiations with the Ministry were ultimately unfruitful. In 1936, during the period of the Front populaire, engineers worked to be included in the negotiations on collective conventions, with the union having to exclude employers from its membership. In spite of their differences and quarrels, the different unions of engineers came together to form a national Federation of engineers unions in order to have weight as a significant social partner.

\section{Keywords}

Engineers unemployment. Social conventions. Chemical engineer diploma. State diploma. Chemistry training. Gunpowder engineers. Foreign engineers. Union of French Chemical Engineers (SIFC). Federation of French Engineers Unions (USIF). Chemical Industries Union (UIC). 


\section{Introduction}

Le texte présenté ici revêt une facture particulière. Il se fonde essentiellement sur une analyse du bulletin d'information du Syndicat professionnel des ingénieurs chimistes de France, L'ingénieur chimiste, revue en principe mensuelle, qui parait durant l'entre-deuxguerres. Il s'agit donc de rendre compte, d'un point de vue « indigène », des préoccupations qui agitent ce groupement, des campagnes qu'il lance, des combats qu'il entend mener, des polémiques qui l'agitent. Le bulletin présente également un état de l'organisation à différents moments de son existence, il signale aussi les ouvrages qui peuvent intéresser professionnellement ses lecteurs. $A$ contrario, certaines thématiques mobilisant en principe le milieu des chimistes ne sont pas traitées ou sont à peine mentionnées, par exemple la question des brevets ou, plus largement, la place de la recherche scientifique. En redonnant ainsi la parole à un groupe qui cherche alors à assurer son identité spécifique, au sein de la couche segmentée si ce n'est fragmentée des ingénieurs de l'époque, on vise à rendre compte des représentations, des élans, des aspirations, sinon des illusions d'une couche professionnelle qui veut d'autant plus s'affirmer qu'elle se perçoit comme trop peu considérée, voire mal aimée du monde industriel. Au-delà de leurs soucis propres, les chimistes participent bien des inquiétudes d'une catégorie professionnelle en devenir, celle des ingénieurs, qui, d'une part, entend asseoir sa position professionnelle au sein de l'entreprise, encore insuffisamment assurée - notamment par la reconnaissance du diplôme - mais dont, d'autre part, le statut social reste fragile, la 
crainte du déclassement constituant une hantise constante, d'où leur participation à des mouvements de défense et de promotion des classes moyennes.

\section{Naissance, organisation et objectifs du syndicat}

La fin de la Première guerre mondiale voit naître une floraison de syndicats d'ingénieurs. C'est un phénomène nouveau par son ampleur et par sa nature. Le fait syndical avait été officialisé par la loi en 1884 et c'est le mouvement ouvrier qui s'était d'abord tout naturellement saisi de cette opportunité. Bien que quelques structures syndicales non ouvrières se fussent constituées avant-guerre, comme par exemple une fédération syndicale patronale chrétienne dès 1899, ou la Chambre syndicale des ingénieurs en 1914, leur faible ampleur, les problématiques qu'elles avaient développées les feraient plutôt désigner comme du «proto-syndicalisme ». Même une organisation comme l'USIC, l'Union sociale d'ingénieurs catholiques, qui avait pris la forme syndicale dès 1906, adhérait alors plus à ce statut par opportunité comme modalité fonctionnelle de gestion que pour mettre en œuvre un programme revendicatif portant les demandes d'un groupe professionnel. Les choses changent avec le conflit. Comme l'expliquent clairement Marc Descotes et Jean-Louis Robert, la guerre de 1914-1918 et l'immédiat après-guerre constituent une période décisive pour l'organisation des ingénieurs et des techniciens. « $L a$ guerre amène l'apparition d'un ensemble de faits économiques, sociaux et politiques de grande portée $»^{1}$. Ils pointent ainsi le

\footnotetext{
${ }^{1}$ Descotes Marc et Robert Jean-Louis (dir.), Clés pour une histoire du syndicalisme cadre, Les Éditions ouvrières, Paris, 1984, p. 57.
} 
développement d'une économie de guerre avec la production massive d'armements qui conduit les ingénieurs à prendre de lourdes responsabilités, avec le sentiment «qu'on ne les payait pas en retour $»^{2}$, se sentant lésés par rapport à ceux que l'opinion désignera comme «les profiteurs de guerre », ce qui les amène à prendre conscience d'une opposition à un certain patronat. Le conservatisme de chefs d'entreprise par rapport à l'organisation scientifique du travail mise en œuvre par les ingénieurs peut également les contrarier. La puissance prise par la CGT à l'issue du conflit est un autre facteur explicatif: elle se fait au bénéfice des seuls ouvriers et inquiète les catégories d'encadrement, car les actions syndicales allant jusqu'aux grèves, mettent à mal leur autorité. Entre le patronat et les couches ouvrières,

«on conçoit [...] qu'il y ait eu place pour toute une gamme d'attitudes très diversifiées relevant pour la plupart de la recherche de la constitution d'une troisième force, celle de la technique, de l'intelligence entre le capital et le travail ${ }^{3}$.

Cependant, ces auteurs n'examinent pas du tout les motifs de constitution de groupements revendicatifs d'ingénieurs liés directement à ce qui avait été vécu au Front, pendant les quatre années de guerre. C'est pourtant une thématique qui sera largement exposée dans la revue depuis son premier numéro en décembre 1919.

En tout cas, c'est pendant la guerre que des groupements de chimistes se seraient formés en divers points du territoire. C'est ainsi qu'on situe, dès le $1^{\text {er }}$ numéro de L'ingénieur chimiste, l'origine du

\footnotetext{
${ }^{2}$ Ibid

${ }^{3}$ Ibid., p. 58.
} 
Syndicat professionnel des ingénieurs chimistes français (SICF). Le texte n'en dit pas plus, on ne saura donc pas en fonction de quels mots d'ordre, en raison de quelle opportunité, et dans quelles régions ou quelles villes, des chimistes ont jugé utile de se regrouper. Mais revenant sur les conditions de cette création, l'organe du nouveau syndicat indique que dès le 9 février 1919 était organisée une réunion dont l'objet était de définir la fonction d'un syndicat d'ingénieurs chimistes «qualifiés». Les participants étaient vraisemblablement des membres de l'Union des associations d'anciens élèves des écoles de chimie (UNADEC) créée en 1910 par d'anciens élèves de l'École de physique et de chimie industrielles de la Ville de Paris (EPCI) qui avaient pris l'initiative d'y faire adhérer les anciens des instituts universitaires de chimie ${ }^{4}$. C'est du reste l'Union qui a rédigé le projet de statuts. Dans la volonté de rassembler l'ensemble des chimistes professionnels, un contact est très vite pris avec la Société des chimistes français pour proposer à celle-ci un regroupement ${ }^{5}$. L'accueil est de prime abord favorable. Cependant, tout le monde n'est pas d'accord dans la Société, le conseil d'administration vote et une majorité apparaît pour refuser de s'associer au projet et se transformer en structure syndicale. Devant cette opposition, les promoteurs de l'opération renoncent à la fusion, et ils s'organisent. Le syndicat est créé le 23 mai 1919. Le 25 mai, une assemblée de fondation est réunie et un conseil d'administration provisoire est

\footnotetext{
${ }^{4}$ Je remercie Nathalie Hugot-Piron qui m'a aimablement fourni ces renseignements.

${ }^{5}$ La Société des chimistes français est née en 1917 de la transformation du Syndicat central des chimistes et essayeurs de France, créé en 1890 - six ans après le vote de la loi autorisant la création de syndicats. La Société finit par fusionner avec le SICF. Les informations proviennent de la nécrologie du fondateur, le pharmacien Calixte Crinon, décédé à 90 ans, publiée dans le n81 de juillet 1929 du bulletin.
} 
nommé. Émile Fleurent, ingénieur EPCI, professeur au Conservatoire des arts et métiers, un personnage important du monde chimique ${ }^{6}$, est désigné comme président et dans le conseil, on trouve des représentants des écoles et instituts de Lyon, Paris, Lille, Nancy, Bordeaux, Toulouse et Mulhouse. Parallèlement se créent au même moment un syndicat des ingénieurs électriciens et un syndicat des ingénieurs mécaniciens dont les statuts sont similaires. D’entrée, il est prévu une fédération de ces trois syndicats ${ }^{7}$. Ce sera 1'Union des syndicats d'ingénieurs français (USIF) qui jouera durant tout l'entredeux-guerres un rôle important d'animation de ce milieu.

Pourquoi un tel syndicat? Les patrons d'un côté, les travailleurs manuels de l'autre ont su se regrouper mais pas les classes intermédiaires. Des groupements de chimistes existent déjà mais ils ont un caractère amical ou technique ou industriel : "aucun ne se consacre exclusivement à l'action corporative. Il s'agit de donner leur véritable place aux chimistes dans les hiérarchies de l'industrie, de l'enseignement public et des services publics $»^{8}$. Ce sera désormais une thématique constante du syndicat: il faut revaloriser le statut et l'image du chimiste. Autre thématique forte, le libre accès aux postes de l'État. C'est une revendication ancienne chez les ingénieurs civils : elle motivait déjà les fondateurs de la Société des ingénieurs civils de France en 1848 qui voulaient obtenir l'accès au corps des ingénieurs des Ponts et Chaussées en même temps qu'ils dénonçaient le

\footnotetext{
${ }^{6}$ Voir à son propos, sa notice biographique rédigée par Gérard Emptoz, pour le Dictionnaire des professeurs du Conservatoire national des arts et métiers, CNAM et INRP, Paris, 1994, tome 1, p. 504-514.

${ }^{7}$ Cf. Descotes Marc, Robert Jean-Louis (dir.), op.cit. l’USIF, p. 66-73.

${ }^{8}$ L'Ingénieur chimiste [désormais I.C.], n 1, décembre 1919.
} 
monopole de ce corps sur un ensemble de travaux publics. 70 ans plus tard, c'est la même complainte.

Les principales revendications énoncées sont donc les suivantes :

"Reconnaissance officielle du titre d'ingénieur-chimiste par l'État et mise de ce titre sur le même pied que les diplômes des grandes écoles

Libre accès à tous les concours et à tous les postes de l'État qui nécessitent des techniciens de la chimie

Faire partie des différents conseils économiques nationaux et régionaux

Obtenir un statut militaire spécial de la même manière que les élèves des grandes écoles

Régénérer l'enseignement de la chimie industrielle par :

L'unification des programmes des examens d'entrée et des étudiants pour les différents instituts et écoles

L'introduction de techniciens qualifiés donnant un enseignement aussi pratique que possible, dans le personnel enseignant

L'admission de techniciens anciens élèves de préférence dans les conseils de perfectionnement de chaque école " $"$.

Curieusement, aucun des items de ce premier programme d'action ne concerne l'activité professionnelle dans le secteur industriel, et notamment la question des rémunérations. Les exigences exposées touchent à l'identité du chimiste et visent en premier lieu à une reconnaissance «officielle» de celle-ci par l'État, d'abord en considérant la qualification intrinsèque du chimiste justifiée par son diplôme, ensuite en obtenant l'égale dignité de statut avec les diplômés des grandes écoles, c'est-à-dire essentiellement avec les polytechniciens, aussi bien pour les recrutements dans les services publics que dans le cadre d'une incorporation dans l'Armée,

\footnotetext{
${ }^{9}$ I.C., ${ }^{\circ} 4$, mars 1920.
} 
éventuellement en cas de mobilisation, enfin en accédant ès qualité aux organismes de réflexion économique ${ }^{10}$. Ces préoccupations morales revêtent une très grande importance pour les chimistes, elles seront réaffirmées sous une forme ou une autre de façon régulière dans le bulletin. Pourtant, le cinquième et dernier point vient, en quelque sorte, contredire les précédents, en soulignant que la formation, telle qu'elle existe à ce moment, ne correspond précisément pas aux objectifs du groupe : on peut lire entre les lignes que celle-ci est hétérogène et diffère en qualité selon les établissements, que l'enseignement est trop théorique, car il est sous la responsabilité de formateurs non professionnels, et que pour pallier ces insuffisances, la présence des chimistes professionnels dans les instances des institutions de formation est indispensable. Cette question de la formation sera, durant toute la période considérée, un des axes majeurs de l'action du syndicat.

Qui recruter puisqu'il s'agit de rassembler des chimistes «qualifiés»? Le règlement intérieur détermine quatre catégories dans lesquelles les chimistes souhaitant adhérer pouvaient entrer : on différencie ceux qui ont été formés dans des institutions spécialisées de ceux qui sont arrivés plus tardivement à la chimie; ces derniers doivent avoir une pratique industrielle déterminée. Les premiers dans l'ordre sont les anciens élèves des instituts et écoles de chimie universitaires, mais on y trouve aussi les centraux ayant suivi la spécialité chimie. Les seconds sont issus «des écoles officielles préparant aux carrières industrielles » à savoir Centrale,

\footnotetext{
${ }^{10}$ C'est la « reconnaissance juridique », un des trois types de reconnaissance sociale proposés par le philosophe Axel Honneth. Honneth Axel, La lutte pour la reconnaissance, Cerf, Paris, 2000.
} 
Polytechnique, les Mines de Paris et de Saint-Étienne, l'Institut industriel du Nord, ainsi que les pharmaciens diplômés, les licenciés et docteurs ès sciences et les anciens élèves (non diplômés) des instituts et écoles de chimie ayant accompli toute leur scolarité : ils doivent justifier trois ans de pratique. La $3^{e}$ catégorie regroupe les anciens élèves d'écoles pratiques ou spéciales devenus chimistes: on $\mathrm{y}$ compte l'École des sciences de Rouen, l'École centrale de Lyon (qui n'est donc pas mise au même niveau que celle de Paris), les écoles d'agriculture, et les élèves issus des laboratoires du Muséum ou du CNAM. Il est exigé pour ceux-ci au moins six ans de pratique dont trois de direction technique ou de responsabilité effective. Enfin un dernier ensemble réunit les chimistes qui n'appartiennent à aucune des catégories précédentes. De fait, il vise les autodidactes, encore très nombreux durant cette période : ceux-là doivent prouver dix ans de pratique dont trois de direction technique ou de responsabilité effective. Il s'agit bien de recruter des ingénieurs chimistes, qu'ils en aient le titre effectif ou les fonctions en entreprise, et non des agents techniques, aides de laboratoire ou ouvriers professionnels même très qualifiés.

La structuration du syndicat est prévue selon deux modalités combinées. D'une part, il s'agira de constituer des sections professionnelles au nombre de sept, essentiellement organisées en fonction du type de production industrielle: 1. Grosse industrie chimique, acides, engrais, soudières, poudres, électrochimie ; 2. Produits chimiques purs, pharmaceutiques, photographiques; 3 . Matières colorantes, teintures, parfums et produits plastiques; 4. Caoutchouc, corps gras, couleurs et vernis; 5. Combustibles, 
métallurgie, électrométallurgie; 6. Produits agricoles, industrie des fermentations, cuirs, peaux, colles ; 7. Catégorie résiduelle ou l'on retrouve l'enseignement technique, les labos de recherche, les chimistes-conseils et experts. D'autre part, il faut structurer le syndicat au niveau régional. D'entrée sont annoncées les villes où il existe une tradition forte d'industrie chimique : Bordeaux, Lille, Lyon, Nancy, Rouen. D'autres viendront s'ajouter ensuite comme Strasbourg, SaintÉtienne ou Marseille.

Le syndicat veut s'organiser rapidement. Il dit compter immédiatement quelque 600 membres, mais il a vocation à rassembler tous les chimistes industriels. Au plus fort de son activité, dans les années 1930, il indiquera rassembler 2500 membres, mais en moyenne, durant cette décennie, il comptera plutôt 2000 adhérents, ce qui correspondrait à la moitié des ingénieurs chimistes en fonction en France à l'époque ${ }^{11}$. Selon Descotes et Robert, il regrouperait 50\% des ingénieurs de l'USIF $^{12}$. Le même ouvrage chiffre la population des ingénieurs électriciens syndiqués à 1500, ce qui correspondrait au tiers des ingénieurs de l'Union syndicale. Le troisième syndicat qui compose l'Union des syndicats d'ingénieurs français, créée officiellement le 11 novembre 1919, est celui des ingénieurs de la mécanique, de la métallurgie et des travaux publics qui restera tout du long de son existence une petite structure de 4 à 500 membres. Les ingénieurs sont relativement peu nombreux à l'époque, sans doute autour de 50 à 60 000. Face à la CGT et à la Confédération patronale,

${ }^{11}$ Référence relevée dans Bureau international du travail, «Les conditions de vie des ingénieurs et des chimistes », Études et documents, Série L (travailleurs intellectuels) $n^{\circ} 1$, Genève, 1924. p. 46.

${ }^{12}$ Descotes M. et Robert J.-L., op.cit., note 1, p. 66. 
ils pèsent peu. C'est pourquoi les fondateurs voudraient constituer une Fédération des techniciens de l'industrie française, qui ne verra jamais le jour sous cette forme. Ce sont les événements de 1936 qui pousseront à un regroupement des composantes syndicales d'ingénieurs, mais dans un tout autre contexte. En revanche, l'idée de mobiliser l'ensemble des travailleurs intellectuels, l'élite de la classe moyenne, se fait jour très rapidement. De fait, elle sera créée en mars 1920, sous le nom de Confédération des travailleurs intellectuels, certes très hétérogène dans sa composition, mais beaucoup plus imposante. Les chimistes y seront représentés par l’USIF.

\section{La guerre et le fait syndical}

Les chimistes ont très mal vécu la période de la guerre. Alors qu'ils estiment que le nombre de morts chimistes a été au minimum $15 \%$ de l'effectif mobilisé, ils ont fait l'analyse qu'ils étaient déconsidérés, méprisés, non utilisés pour leurs compétences. Un débat en janvier 1920 à la section lyonnaise l'expose sans ambages. On rappelle leur situation lamentable pendant la guerre : au début, les 9/10 ${ }^{\mathrm{e}}$ ont été mobilisés aux Armées ou dans des postes qui n'avaient aucun rapport avec leur formation scientifique.

«Si par la suite, les services du Front et de l'Arrière se décidèrent à utiliser quelques chimistes, ce ne fut jamais que sans mandat officiel, sans avancement ni grade, sans aucune espèce de rémunération raisonnable. Les ingénieurs-chimistes les plus experts se trouvèrent placés sous les ordres de pseudochimistes qui tiraient leur avantage non de leur savoir mais de leur grade ou de leur origine plus officielle ${ }^{13}$.

${ }^{13}$ I. C., nº 4 , mars 1920. 


\section{À l'appui de ces constats navrants, des témoignages cruellement cocasses sont régulièrement publiés.}

\section{Le détachement des chimistes}

$\mathrm{Au}$ cours de la guerre, ce chimiste est affecté au « Détachement des chimistes ». "Je me rendis donc au camp de...et fut versé au Détachement des chimistes. Il était assez singulièrement composé. Il y avait là des comptables, des camionneurs, des ouvriers de diverses usines, mais tout ce monde était évidemment dit "chimiste 》 puisque les magasins ou les usines où ils travaillaient en temps de paix se rattachaient d'une manière quelconque à la droguerie ou aux produits chimiques. Il y avait pourtant un des intéressés qui avait des rapports plus éloignés avec la chimie : il était tueur à l'abattoir. Sans doute avait-il été mis là pour représenter la chimie biologique. Il y avait aussi, en me comptant, trois chimistes. Le détachement étant composé de trente hommes, ça faisait juste du 10\%. C'était une proportion très honorable ». Il est question de fabrications d'obus spéciaux. Arrivé sur le lieu, il s'agit plus simplement de déplacer d'un endroit à un autre des obus de 75. D'autres sont chargés de briser à coup de massue de vieux obus de 138. Ils doivent subir les encouragements du chef de laboratoire (!), un adjudant de 20 ans de caserne... "Le grand ouvre se préparait pendant ce temps. Au bout de quelques jours en effet, la besogne prit un caractère plus technique... Les obus de $75 \ldots$ devaient recevoir quelques bâtons de phosphore et quelques décilitres de sulfure de carbone. Le travail fut confié aux hommes du Détachement des chimistes. L'un versait dans le corps de l'obus une éprouvette de sulfure de carbone, un deuxième y introduisait d'une main sûre les bâtons de phosphore et comme ceux-ci, d'un diamètre trop gros pour l'œil de l'obus refusaient parfois d'y passer, ils y étaient chassés à force coups de maillet. Un troisième enfin vissait la gaine. Par une visible protection de Sainte Barbe, patronne des artilleurs, j'atteste que pendant les quinze jours que je passais à l'atelier de chargement, il ne se produisit pas d'accident. J'avoue néanmoins que je me défilai lâchement et laissai faire la besogne aux collègues chimistes qui avaient des idées moins arrêtées que les miennes sur ce qu'on peut raisonnablement demander d'incombustibilité au phosphore et au sulfure de carbone. Mes deux camarades chimistes agirent de même, compromettant ainsi en ma compagnie l'honneur de la corporation $»^{14}$.

${ }^{14}$ I.C., n5, 15 avril 1920. Ce témoignage est repris comme argumentaire dans la publication du BIT de 1924 (op. cit.), en exposant que le titre de chimiste n'est pas efficacement protégé par la loi, car pendant la guerre, l'administration militaire « lui a donné un sens que les ingénieurs-chimistes ont considéré comme nuisible à leurs intérêts ». p.31. 
L'ire des chimistes se focalise particulièrement sur le corps des ingénieurs des Poudres qu'ils poursuivent de leur vindicte dans de nombreux numéros de la revue. On leur attribue généralement une incapacité fondamentale à faire de la chimie moderne ("ils ne savent faire que de la poudre noire $»^{15}$ ) alliée à une acuité remarquable pour interdire l'accès à leur corporation des «véritables » chimistes. Le sentiment général est celui d'un véritable gâchis.

\section{Mes campagnes}

(...) «Tout de même, on a quelques joies pures. Apprendre, par exemple, que pour blanchir les cotons récalcitrants on a commandé 20 tonnes de bicarbonate de soude parce que 'le sel Solvay n'est pas assez fort' (naturellement du bicarbonate doit être rudement plus fort que du simple carbonate!). Répondre, la face impassible, tandis qu'on jubile intérieurement: 'je ne sais pas' à un ingénieur à quatre galons qui vous demande froidement quelles matières colorantes on emploie pour faire les rayons ultraviolets. Entendre un ingénieur de $2^{e}$ classe vous demander du papier de tournesol pour voir 'si le bisulfate de soude est bien neutre' - tout ça, ça fait passer de bons moments! Mais il y a aussi de fichus quarts d'heure! » (Extrait de mon carnet de guerre du 6 avril 1916) ${ }^{16}$.

«Le projet de MM. Paté et Breton créant un cadre d'ingénieurs chimistes militaires déposé à l'instigation de l'Union ne peut venir en discussion et lors de l'application de la loi Mourier, les chimistes furent assimilés à de simples manœuvres jusqu'à l'institution, en 1918 de la Commission spéciale demandée par l'Union nationale ${ }^{17}$.

${ }^{15}$ I.C., n7, juin 1920. Rappelons tout de même que Paul Vieille, ingénieur des Poudres (1884-1934), a conçu et développé la poudre blanche (poudre B) ou poudre sans fumée, en 1884 !

${ }^{16}$ I.C., $\mathrm{n}^{\circ} 10$, septembre 1920.

${ }^{17}$ I.C., n5-6, mai 1920. Il s'agit ici de l'Union nationale des associations d'anciens élèves des écoles de chimie (UNADEC). La loi du 27 février 1917, dite loi Mourier, vise à réaffecter des exemptés ou réformés dans les services actifs ou auxiliaires de l'Armée, sous réserve d'un contrôle médical. 
On émet le vœu que le recensement des chimistes entrepris par la Commission soit continué afin de régler leur affectation et que les écoles et instituts de chimie soient dotés d'un régime militaire analogue à celui de l'École centrale. Dans cette période du début des années 1920, les souvenirs sont encore vifs et le syndicat se préoccupe de tirer un bilan pour éviter à l'avenir que de semblables dysfonctionnements se reproduisent. Il faut repenser la législation et la réglementation pour qu'à la prochaine mobilisation, on puisse incorporer les chimistes dans des positions militaires où ils pourront rendre véritablement service au pays. Néanmoins, les membres du syndicat qui s'expriment, sont dubitatifs et la hargne reste tenace.

\section{"Ils " ne changeront jamais}

"Certains optimistes supposaient que la guerre avait appris à l'administration militaire l'existence du chimiste (...) Cet abandon des plus respectables traditions militaires était due à ce que l'armée, pendant la guerre, s'était, si j'ose dire, civilisée. La paix est revenue, l'armée est redevenue militaire et les chimistes sont maintenant replacés à leur vrai rang : le dernier ».

L'administration de la Marine monte un laboratoire des poudres et explosifs de l'artillerie navale. L'officier chef de service a besoin de chimistes et s'adresse au service de placement d'une association d'anciens élèves. Quels seraient les appointements ? Un minimum de traitement de 800f/mois pour un chimiste débutant. Le représentant de l'association indique " qu'il fallait cesser de parler de salaires et d'ouvriers et qu'il ne pouvait être question que d'appointements d'ingénieurs chimistes - Mais administrativement, il n'y a pas d'ingénieurs chimistes, s'exclama mon interlocuteur! Le titre d'ingénieur est assuré dans le corps de la Marine aux seuls ingénieurs issus d'où vous savez, il ne peut y en avoir d'autres! Les chimistes ne peuvent être que des ouvriers! - Allez dire à ceux qui vous envoient que chez nous, nous ne tenons pas cet article, nous ne savons pas ce que c'est, nous ne connaissons que des Ingénieurs-chimistes (...) $»^{18}$.

\footnotetext{
${ }^{18}$ I.C., nº8-9, juillet-août 1920.
} 
Par ailleurs, le syndicat rappelle que la mobilisation n'avait pas prévu la nécessité d'assurer la fabrication des poudres et explosifs. «Cette erreur qui faillit être funeste à notre pays, a retardé la victoire $\gg{ }^{19}$. Pourtant, si les témoignages sont nombreux sur ce qui se passait sur le Front, le bulletin livre très peu d'information sur l'arrière, le « second Front $»^{20}$, alors que du fait du prolongement de la guerre, il a fallu procéder à l'extension des établissements chimiques existants et ouvrir de nouvelles usines. Dans un exposé présenté en 1920 sur l'importance de la production en chimie de guerre pendant le conflit, Albin Haller conclut sur l'augmentation considérable du personnel affecté à cette tâche :

"Le personnel nécessaire a été d'un recrutement difficile pendant la guerre. En juillet 1914, le Service des Poudres comptait 7700 personnes dont 44 ingénieurs; il en comptait 120000 à la fin de 1917. Ce sont les chimistes qui ont le plus manqué $»^{21}$.

Pourquoi cette dimension importante du travail des chimistes est-elle à ce point minorée? En premier lieu, sans doute, parce que les techniciens situés à l'arrière, s'ils ont contribué à la victoire en produisant les outils et matières nécessaires aux combattants, n'ont

${ }^{19}$ I.C., $\mathrm{n}^{\circ} 5-6$, mai 1920.

${ }^{20}$ Fridenson Patrick (dir.), 1914-1918, l'autre front, Les Éditions Ouvrières, Paris, 1977.

${ }^{21}$ Bulletin de la Société d'encouragement à l'Industrie nationale, mai-juin 1920, tome 132, séance publique du 15 mai 1920, p. 385. http://cnum.cnam.fr/CGI/fpage.cgi?BSPI.134/390/100/1025/0/0

De son côté, L'Ingénieur-Chimiste rapporte un discours du savant, alors directeur de l'EPCI, revenant sur la guerre qui vient de s'achever, lors d'une commémoration : "Hélas, cette armée n'était cependant qu'en puissance tant fut grande l'imprévoyance de nos Chambres, et primitive [sic] les services d'ordre technique de notre administration militaire où, sauf quelques rares exceptions, on paraissait ignorer la part importante qui reviendrait à l'industrie de l'arrière dans la lutte gigantesque prolongée qui allait s'engager». I.C., n², janvier 1920. 
fait que leur travail habituel au sein des entreprises. Il est logique qu'à la sortie de la guerre, le rôle des combattants soit valorisé, exalté. Il témoigne avec vigueur du patriotisme des chimistes enrôlés, ce qui constitue un motif légitime de fierté pour l'ensemble de la corporation. On peut aussi poser l'hypothèse selon laquelle le jeune syndicat doit, pour mobiliser ses troupes, exposer une injustice flagrante : comment, face à cet héroïsme, la non prise en considération des compétences des chimistes n'a pas permis de les utiliser efficacement à leur juste valeur pour le bien de la patrie. Les coupables sont désignés : ce sont les polytechniciens du Service des Poudres, au sommet de la hiérarchie militaire, qui n'emploient les chimistes engagés qu'à des tâches subalternes, ce qui revient à leur dénier toute qualification ${ }^{22}$. Cette déconsidération est vécue collectivement comme une humiliation et l'un des axes du syndicat sera précisément de militer pour la reconnaissance de leurs connaissances scientifiques et de leur expertise ${ }^{23}$.

${ }^{22}$ Le cas le plus célèbre est celui de Victor Grignard, prix Nobel 2012, utilisé la première année du conflit à surveiller les voies ferrées (d'autres biographies le signalent comme garde-côte). Georges Claude pourra plus facilement faire valoir ses compétences. Affecté à l'Artillerie comme sous-lieutenant, il est nommé en août 1914 membre de la Commission supérieure des Inventions où il pourra mettre en œuvre ses inventions en matière d'explosifs. Voir : Baillot (Rémi), Georges Claude, le génie fourvoyé, EDP Sciences, Paris, 2010, p. 162 sq.

${ }^{23}$ À l'inverse de cette position, il faut noter le point de vue de Louis Hackspill, futur directeur de l'Institut de chimie de Paris; «il est contre l'idée de réserver aux chimistes des " jeunes classes ", c'est-à-dire ceux de moins de trente ans, une situation militaire spéciale. La situation des jeunes chimistes est la même que celle des autres ingénieurs : ils sont plus utiles au front comme officiers. Les aptitudes scientifiques permettent de lire des cartes, de calculer des positions, de dessiner. La supériorité de l'artillerie française lors de la grande guerre vient pour Hackspill de la présence des ingénieurs des arts et manufactures au front. Il n'est donc pas utile d'affecter des ingénieurs chimistes dans les poudreries, les laboratoires et les ateliers de chargement d'obus, ces postes pouvant être occupés par des manœuvres et des femmes ». Voir: Le Meur Laurent, Quand la chimie industrielle croise la chimie 
Toutefois, des motifs plus amples sont également mis en avant pour expliciter l'émergence d'une réflexion collective, pendant la guerre et dans l'immédiat après-guerre, conduisant à des regroupements syndicaux. Un article d'avril 1920 expose cette analyse qui dépasse le seul cadre de la chimie pour englober l'ensemble des ingénieurs.

"... La guerre a provoqué le développement intensif de toute la technicité. (...). Le rôle de l'ingénieur, naguère ignoré, méconnu, quelquefois même déconsidéré, a été mis en lumière aux yeux de tous, et c'est aujourd'hui sans aucun étonnement que l'opinion publique voit se réunir en groupements syndicaux ceux qui furent par la subtilité de leur pensée inventive, par leur énergie d'organisation directive, les artisans du glorieux succès de notre armée.

Le syndicalisme des ingénieurs (...) est né au cours de l'accomplissement du labeur sacré de la Défense nationale. Il semble qu'au rude contact des impitoyables nécessités de la vie d'usine et de laboratoire pendant la guerre, il ait acquis, à ses débuts, un caractère spécifique fait de la notion acquise du droit corporatif uni à la conception d'un devoir professionnel nettement orienté vers le service de la collectivité ».

Plus loin, on peut lire ces remarques :

"Les techniciens sont au centre de l'organisation économique. Ils ont pour mission d'assurer la production, d'en modifier utilement le mécanisme, d'améliorer le rendement des entreprises, d'utiliser rationnellement la main d'œuvre. La condition du travailleur ne peut s'élever que grâce aux découvertes techniques de l'ingénieur. La prospérité nationale ne peut augmenter qu'à la suite de transformations du même ordre, guidées par la science qui, seule, possède un pouvoir créateur de richesse. (...).

minérale. Louis Hackspill (1880-1963). Mémoire de master 2, Centre François Viète, Nantes, 2009-2010, p. 72. Toutefois, la plupart des diplômés des instituts annexes des facultés des sciences n'étaient pas considérés comme ingénieurs et classés comme tels par l'administration militaire. 
De tous les phénomènes sociaux provoqués par la guerre, il est un des plus importants (...) celui qui présente le caractère de plus grande nouveauté dans la formation et le fonctionnement $»^{24}$.

$\mathrm{Du}$ reste, rapidement, les revendications prennent forme. À la différence des travaux des associations d'anciens élèves, d'où les chimistes du SICF sont très largement issus, il ne s'agit pas d'émettre des vœux, mais de constituer des revendications crédibles et d'entreprendre des négociations. C'est donc un « vrai » syndicat qui se constitue, porteur des attentes et demandes de ses membres. La base minimale émise à la création du syndicat va s'étoffer rapidement. Le premier point est classiquement celui des salaires.

\section{La question des salaires (les « appointements ${ }^{25}$ )}

Une première proposition est émise lors du premier conseil d'administration du syndicat en mai 1919: une commission approuvée par le CA propose un minimum de $600 \mathrm{f} / \mathrm{mois}$ pour les chimistes sortis d'une école ou d'un institut. Il est fait appel aux patrons chimistes pour qu'ils respectent cette règle en engageant des jeunes chimistes. Cette somme correspondrait à un salaire mensuel de 200 f. en 1914, un peu supérieur à celui d'un ouvrier non spécialisé,

\footnotetext{
${ }^{24}$ I.C., n ${ }^{\circ} 5$, avril 1920.

${ }^{25}$ Les ingénieurs, et d'une façon générale les professionnels, veulent se distinguer des ouvriers et des employés inférieurs qui touchent des "salaires ». La distinction porte sur le terme utilisé. On parlera d'appointements qui supposent un versement régulier mensualisé. C'est, semble-t-il, en 1936 avec les négociations sur les conventions collectives que les termes salaire et salariat se généraliseront. Les avenants cadres des conventions collectives en 1945, connues sous l'appellation arrêtés Parodi (du nom du ministre du Travail de l'époque), confirmeront cette évolution. Symboliquement, les ingénieurs et cadres convenaient alors que le contrat de travail qui les subordonnait à un patron les instituait salariés.
} 
équivalent à un salaire moyen d'un ouvrier spécialisé, un peu inférieur à celui offert par les services de l'État ou de la ville de Paris. Il est alors recommandé aux chimistes qui se placent, qu'ils refusent des salaires inférieurs ${ }^{26}$.

On notera la modestie de la revendication initiale. Rapidement une commission du statut revient sur ce point, jugeant insuffisante la proposition de $600 \mathrm{f}$. Provisoirement, elle fixe la somme de $750 \mathrm{f}^{27}$. Elle envoie un questionnaire auquel répondent 222 adhérents (sur à peu près 600 , à ce moment là). Le tiers des répondants gagne moins de $1275 \mathrm{f} / \mathrm{mois}^{28}, 20 \%$ reçoivent entre 1275 et $1625 \mathrm{f} / \mathrm{mois}$... Encore faut-il moduler entre les régions, ceux de Lyon gagnent significativement moins que la moyenne nationale, et ceux de la région parisienne un peu plus. On convient d'un principe général : un ingénieur ne peut en aucun cas être moins rémunéré que ceux qui normalement travaillent sous ses ordres ${ }^{29}$.

En novembre 1920, la revue publie le travail d'un ingénieur du syndicat frère des chimistes, celui des ingénieurs électriciens, sur les traitements de début d'un ingénieur ${ }^{30}$. Il dresse une liste extraordinairement détaillée des dépenses de cet individu, depuis l'habillement complet, l'entretien du corps, le blanchissage, le raccommodage, le logement, la nourriture, etc. jusqu'à la cotisation au syndicat et les banquets d'amicale, auquel il ajoute les impôts, un

\footnotetext{
${ }^{26}$ I.C., $\mathrm{n}^{\circ} 1$, décembre 1919.

${ }^{27}$ I.C., ${ }^{\circ} 5 / 6$, mai 1920 .

${ }^{28}$ Il ne s'agit pas forcément de débutants...

${ }^{29}$ I.C., ${ }^{\circ} 7$, juin 1920 .

${ }^{30}$ I.C., $\mathrm{n}^{\circ} 12$, novembre 1920.
} 
voyage annuel, des frais de théâtre et imprévus. Il parvient à une somme de $833 \mathrm{f} /$ mois qu'il compare avec les émoluments de militaires de carrière (sous-lieutenant, lieutenant et capitaine) : le salaire du chimiste est manifestement inférieur et de beaucoup. Le mois suivant, un long article du président Émile Fleurent revient sur la question :

"Avant 1914, dans la grande majorité des cas, les salaires de début offerts aux chimistes capables, par leur origine et leurs connaissances pratiques acquises au laboratoire, de remplir le rôle d'ingénieurs, correspondaient à peine aux conditions matérielles de l'existence. (...) En ce moment (...) les conditions se sont profondément modifiées dans le sens d'une aggravation à laquelle doit correspondre, au minimum, une augmentation du salaire.

(...) Les conditions morales doivent également entrer en ligne de compte, le technicien, par l'éducation que lui confère l'instruction générale et professionnelle qu'il a reçue, aussi bien que par cette instruction même, possédant une supériorité intellectuelle qui doit être nettement affirmée, vis-à-vis du personnel qu'il aura ou pourra avoir sous ses ordres, dans l'intérêt même de l'affaire à laquelle il apporte ses services $\gg{ }^{31}$.

Des négociations ont été entreprises rapidement avec le Syndicat général des produits chimiques, sur la base de la proposition d'un minimum de $750 \mathrm{f} / \mathrm{mois}$ avec l'argumentaire suivant : ce montant est le «seul capable d'éviter au jeune ingénieur les soucis de la vie journalière et de la placer ainsi dans toute la plénitude du rendement que l'industriel est en droit d'attendre de lui ». Mais elles ont traîné en raison des transformations de l'organisme patronal en une fédération de syndicats, devenant ainsi l'Union des industries chimiques (UIC). Il faut donc que cette fédération demande un accord de principe à chacun de ses syndicats : dans leur grande majorité,

\footnotetext{
${ }^{31}$ I.C., n 13 , décembre 1920.
} 
ceux-ci approuvent cette proposition. L'UIC va donc transmettre une recommandation à ses adhérents :

«Il a semblé que, d'une façon presque générale, le chiffre de $750 \mathrm{f}$. d'appointements mensuels de début (avantages divers compris) doive être favorablement envisagé pour les ingénieurs chimistes et chimistes donnant les garanties de capacité requises, c'est-à-dire possédant les conditions de scolarité et de stage prévues tant par le Syndicat professionnel des ingénieurs chimistes dans son règlement intérieur pour l'admission de ses membres que par la Société des chimistes français ». C'est signé par son président, René Paul Duchemin. Fleurent remercie chaleureusement le syndicat patronal et «son distingué président que nous comptons parmi nos membres... $»^{32}$.

L'enquête salariale auprès des membres sera renouvelée régulièrement. Elle est confiée à une commission du statut du chimiste et des appointements ${ }^{33}$. Cette enquête sert à alimenter les négociations - qui demeurent toujours d'une extrême courtoisie - avec l'Union des industries chimiques. Ainsi en mai 1929, il est indiqué qu'à la suite de conversations entre le syndicat et l'UIC, celle-ci a adressé à ses

\footnotetext{
32 Ibid. Aucune référence législative n'est invoquée. Cet accord aurait pu s’inscrire dans le cadre de la loi du 25 mars 1919 qui organise pour la première fois les conventions collectives. Les dispositions de cette loi impliquent que la convention collective s'applique même quand elle contredit des éléments du contrat de travail individuel: le collectif prime sur l'individuel. Elle donne aux organisations signataires le droit d'ester en justice pour faire appliquer la convention. Cette première législation sera renforcée et complétée par la loi du 24 juin 1936. Voir notamment: Gazier Albert, "Le retour aux conventions collectives", Revue économique, 1/2 (1950), p. 157-167. Mais comme l'indique le rapport du BIT sur les ingénieurs et les chimistes (op. cit.) très largement fondé pour la France sur les analyses de l'USIF, cette Union avait préconisé un salaire de $600 \mathrm{f}$ pour un ingénieur débutant en 1919, porté à 750f. en 1921. «Mais les syndicats patronaux ne l'ont jamais accepté. Ils se sont refusés à mettre sur un pied d'égalité absolue, à leur sortie de l'école, les élèves des écoles officielles et ceux d'écoles de moindre valeur ». p. 71-72. L'accord entre les chimistes et l'UIC s'assimile donc à un gentleman's agreement.

${ }^{33}$ I.C., n³9, octobre $1925 ; n^{\circ} 76$, février $1929 ; n^{\circ} 84$, novembre 1929.
} 
membres, une circulaire leur recommandant de donner à leurs ingénieurs chimistes, comme minimum d'appointement de début, une somme de1200 f/mois. Cette offre portée à $1300 \mathrm{f}$ après une période d'essais de 6 mois et 1400 après un an. Cette recommandation vaut pour les ingénieurs chimistes tels qu'ils sont définis par les conditions d'admission au syndicat. Suit une liste de 28 syndicats patronaux réunis au sein de l'UIC, concernés par la circulaire ${ }^{34}$.

La question du salaire des ingénieurs ne touche pas seulement les chimistes, mais toutes les branches industrielles. Les explications livrées par le rapport du BIT pour expliquer la faiblesse des émoluments de départ paraissent étranges :

«Cela provient de ce que les ingénieurs font souvent des besognes qui n'exigent nullement leurs compétences techniques. On paie alors le travail qu'ils font et non celui qu'ils pourraient faire. Les syndicats ont fait un gros effort pour obtenir la fixation d'un minimum de début. Ils se sont heurtés à un écueil de principe : s'il s'agit d'un chiffre basé sur le minimum nécessaire à l'existence, il doit être le même pour tous, et les patrons se refusent à accepter de placer sur le même pied les capables et les incapables, les savants et les ignorants. Et si l'on veut faire des distinctions basées sur la valeur des diplômes, on ouvre la porte à l'ambition et surtout à la jalousie ${ }^{35}$.

En fait, le principe de recrutement d'un ingénieur dans une entreprise repose sur le fait que le nouveau venu commence au bas de l'échelle hiérarchique et qu'il endosse le bleu de l'ouvrier, ou, au mieux, la blouse du dessinateur :

\footnotetext{
${ }^{34}$ I.C., ${ }^{\circ} 79$, mai 1929

${ }^{35}$ Ibid., p. 71.
} 
«une formation d'école, quelle que soit sa valeur, ne préjuge aucunement de la capacité à résoudre les problèmes spécifiques qui se posent aux ingénieurs dans l'exercice de leurs fonctions. Le diplome n'est pour l'employeur que le signe encourageant d'une virtualité $\gg 36$.

Ce principe ne touche sans doute qu'à la marge les ingénieurs issus de l'École polytechnique, voire les ingénieurs civils issus des écoles d'application de cette institution, mais tous les autres diplômés, qu'ils soient gadzarts, formés dans les instituts techniques universitaires ou dans d'autres établissements publics ou privés, sont concernés. L'analyse des guides pour l'emploi des carrières, publications très consultées dans l'entre-deux-guerres, confirme la généralité de ce processus. L'exploitation des rares données disponibles concernant les salaires des ingénieurs met en évidence la modestie de départ des rémunérations des ingénieurs, en même temps que les distinctions établies entre des catégories de diplômés, comme le montre l'exemple des compagnies de chemins de fer ${ }^{37}$.

\section{Les causes de la « dépréciation »}

Convaincus que les chimistes français forment un corps professionnel dont le monde politique, les responsables économiques et même la société civile ont une perception dévalorisante de leurs compétences et partant, de leur métier, les syndicalistes proposent

\footnotetext{
${ }^{36}$ Cohen Yves, «Titre d'entreprise contre diplôme d'ingénieur. Les ingénieurs gèrent les ingénieurs ", in Grelon André (dir.), Les ingénieurs de la crise. Titre et profession entre les deux guerres, Éditions de l'École des hautes études en sciences sociales, Paris, 1986, p. 77.

37 Grelon A., «Formation et carrière des ingénieurs en France (1880-1939) », in Bergeron Louis et Bourdelais Patrice (dir.), La France n'est-elle pas douée pour l'industrie?, Belin, Paris, 1998, p. 231-274. Voir notamment le tableau p. 265.
} 
dans leur publication des analyses des causes qu'ils estiment objectives de cette disqualification ${ }^{38}$.

En premier lieu, on considère la situation dans laquelle est tenu l'ingénieur chimiste au sein de l'entreprise. Il est généralement placé dans une position subalterne.

«La vérité, est-il expliqué dans une tribune libre, c'est que les industriels ont besoin de cadres subalternes et pas de cadres supérieurs. On ne forme guère de contremaîtres dans l'industrie chimique, et l'industriel préfère faire tenir ce rôle par un ingénieur chimiste qui se mettra facilement au courant et dont les connaissances étendues donneront plus de garanties que la routine empirique d'un vieil ouvrier $\gg{ }^{39}$.

Les activités auxquelles il est astreint n'exploitent pas ses compétences :

«Les industriels se sont aperçus qu'un laboratoire pouvait servir à quelque chose. Ils se bornent à lui faire faire des analyses de contrôle, toujours les mêmes. On engage pour cela des ingénieurs chimistes payés misérablement qui, dégoûtés $d u$ travail de manouvre qu'on leur fait faire, le considèrent avec négligence et disparaissent à la première occasion. Les industriels maudissent les chimistes et le laboratoire. Pourquoi déclassent-ils un ingénieur à lui faire faire une besogne qui ne peut lui convenir. Il leur faut un bon chef de laboratoire, payé convenablement et des manipulateurs formés facilement avec des ouvriers débrouillards, ou des femmes, non ingénieurs bien entendu $»^{40}$.

\footnotetext{
38 «Dans les industries non exclusivement chimiques, les chimistes sont considérés comme quantité négligeable ou en tout cas, inférieurs aux ingénieurs des autres spécialités ». Allocution à la section lyonnaise, janvier 1920. I.C., nº 4, mars 1920. " Dans l'esprit du public, l'ingénieur sort de Polytechnique, des Mines, des Ponts et Chaussées. Le chimiste fait du laboratoire ». René Sordes, intervention au congrès du 12 février 1928. I.C., nº6, mars 1928.

${ }^{39}$ I.C., n०84, novembre 1929.

${ }^{40}$ Ibid.
} 
Pourquoi les chefs d'industrie ont-ils une telle attitude ? Sans doute parce qu'ils ne sont pas chimistes eux-mêmes. Le constat en est fait par Émile Fleurent qui s'insurge contre le fait que les emplois de direction sont occupés par les élèves des grandes écoles techniques «parce que les affaires industrielles sont tenues par des personnalités qui, par camaraderie, confient la direction des services à leurs 'conscrits' ", à l'inverse de ce qui se fait en Allemagne ${ }^{41}$. Ce constat est repris par l'historien de la chimie Fred Aftalion qui s'est spécialement attaché à l'historiographie de l'industrie chimique. Il éclaire une dimension qu'on ne voit jamais apparaître dans les textes syndicaux des chimistes, à savoir le caractère particulièrement éclaté de la chimie française "très dispersée en une multitude d'unités de petite taille réparties à travers le territoire ", même pour les rares grandes entreprises comme Saint-Gobain avec 25 usines chimiques dont deux seulement atteignent les 900 employés $^{42}$. Il pointe d'autres caractéristiques qui font un contraste singulier avec la concurrence étrangère, notamment allemande. Même s'il souligne les succès d'un Blumenfeld, d'un Georges Claude ou d'un Eugène Schueller, « là où il n'était pas encore nécessaire d'être puissant pour réussir ${ }^{43}$, il insiste sur l'incapacité des dirigeants français à s'entendre pour monter de grandes entreprises performantes, en raison de manques dans l'organisation, les connaissances professionnelles, la continuité dans l'effort, son hypothèse étant que

\footnotetext{
${ }^{41}$ I.C., $\mathrm{n}^{\circ} 11$, octobre 1920.

${ }^{42}$ Aftalion Fred, Histoire de la chimie, Masson, Paris, 1988, p. 188.

${ }^{43}$ Ibid.
} 
«contrairement en particulier aux firmes de la chimie allemande, leurs concurrentes françaises, si l'on excepte Kuhlmann et Rhône-Poulenc, n'avaient pas pour vocation principale le métier de la chimie et n'étaient pas dirigées par des professionnels de cette industrie ${ }^{44}$.

Plutôt que de construire des ensembles cohérents, la volonté des industriels de préserver leur indépendance les conduisait à préférer avant tout des accords de partage, «qui faisaient de l'industrie chimique nationale une mosaïque d'entreprises difficiles à gérer et à rationaliser $»^{45}$. Et de conclure :

«Bien entendu, c'est quand l'importance des moyens à mettre en cuvre devenait prépondérante que les faiblesses de la chimie française, face $\grave{a}$ ses grands concurrents, apparaissaient dans toute leur ampleur, qu'il s'agisse de la recherche, de la technologie, de la taille des unités ou de l'implantation commerciale à l'étranger $\gg{ }^{46}$.

Un tel bilan peut expliquer le manque de valorisation des chimistes employés dans la plupart de ces compagnies industrielles.

Une autre thématique vient compléter l'analyse de la mauvaise posture professionnelle des chimistes français: la concurrence des chimistes étrangers. Cette dénonciation intervient très rapidement dans L'ingénieur-chimiste. Dès le $3^{\mathrm{e}}$ numéro, l'argumentaire est construit. Après avoir pointé la responsabilité des industriels qui, dès avantguerre, recrutaient des chimistes allemands «qui se paraient du titre

\footnotetext{
${ }^{44}$ Ibid. p. 197.

${ }^{45}$ Ibid. La question du non accès des chimistes aux fonctions de direction dans les sociétés françaises de chimie est reprise et démontrée de façon définitive par Hervé Joly, «La position dominée des chimistes à la direction des grandes firmes chimiques en France (années 1900-1960) », in Lamard Pierre et Stoskopf Nicolas (dir.), L'industrie chimique en question, Picard Édition, Paris, 2010, p. 67-87.

${ }^{46}$ Aftalion F., op. cit., note 42, p. 197.
} 
de "Herr Doktor" au détriment des collègues mais qui n'étaient qu'un « nid d'espions », le texte s'attaque aux neutres :

"Pendant la guerre, on a vu trop de chimistes étrangers, des Tchèques, des Suisses, etc. pendant que les collègues mobilisés étaient dans l'incapacité de défendre leurs intérêts. Des personnages dédaigneux, se contentant de regarder marcher l'usine et de passer à la caisse à la fin du mois. Il faut lutter contre la légion des pseudos-chimistes de guerre qui, sous prétexte qu'ils ont été mobilisés dans les poudreries ou les usines travaillant pour la défense nationale grâce à des combinaisons coupables, se font embaucher dans certains laboratoires, ou bien s'installent à leur compte, n'hésitant pas à faire des offres de service sur un papier portant leur nom souligné du titre de chimiste, trompant ainsi par avance la clientèle qui aurait la naïveté de leur confier ses intérêts ${ }^{47}$.

Cette thématique sera reprise tout au long des deux décennies du syndicat. Un premier constat est dressé :

«L'état de guerre a été éminemment favorable à l'établissement, dans nos usines, des chimistes étrangers dont la neutralité permettait toute liberté d'action (...). À l'ouverture de l'état de paix [le chimiste français] a retrouvé ses concurrents étrangers plus solidement établis que jamais, et parfois même dans le poste qu'il avait dîu abandonner au début des hostilités » ${ }^{48}$.

Selon l'auteur de l'article, dans certains établissements, la proportion d'étrangers dans le personnel technique monterait jusqu'à $25 \%$. Pour éviter cette concurrence indue, il faut établir des règles strictes :

\footnotetext{
${ }^{47}$ I.C., n³, février 1920 .

${ }^{48}$ I.C., n¹4, février 1921. Selon Ralph Schor, l'auteur de l'étude sans doute la plus complète sur la perception des étrangers par les Français entre les deux guerres, « les neutres, souvent assimilés à des mercantis qui avaient profité de la guerre pour s'enrichir n'étaient pas beaucoup plus estimés [que les ennemis]». Schor R., L'opinion française et les étrangers en France, 1919-1939, Publications de la Sorbonne, Paris, 1985, p. 69.
} 
"L'emploi d'un chimiste étranger ne paraît justifié que dans un seul cas : la recherche d'une spécialisation bien déterminée introuvable en France, soit parce que la fabrication nouvelle qu'elle intéresse n'a pas été tentée chez nous, soit parce que nos écoles ne se sont pas encore préoccupées de diriger les techniciens vers cette spécialisation ${ }^{49}$.

Dernier élément qui complète l'argumentaire :

«La guerre a révélé à l'opinion publique l'existence et l'importance du chimiste. Est-il admissible qu'une bonne partie de ce personnel, facteur important de notre sécurité nationale, soit composée de techniciens étrangers, si évidente que soit leur neutralité $?^{50} »$.

Dans ces conditions, le syndicat propose l'établissement d'une taxe pour tout industriel employant des chimistes étrangers, envisage de lancer une campagne de presse et offre d'aider le syndicat patronal à trouver des techniciens spécialisés. On mesure que l'organisation qui est alors en négociation avec la fédération patronale pour la détermination des salaires de base, ne peut attaquer frontalement les industriels qui sont pourtant ceux qui ont recruté les «neutres ».

On peut penser que ces inquiétudes sont en partie dues à une poussée du chômage entre 1919 et 1921, liée notamment au retour sur le marché du travail des soldats démobilisés en grand nombre. La situation de l'emploi peut conduire à de telles analyses. On voit précisément une réactivation de la dénonciation des chimistes étrangers pendant la crise des années trente. Les sections régionales alertent sur le fait qu'il y a trop d'étrangers et qu'ils n'ont pas leur

\footnotetext{
${ }^{49}$ I.C., $\mathrm{n}^{\circ} 14$. Ibid.

${ }^{50}$ I.C., Ibid. Selon Schor, l'idée commune était que «ces individus tapis à l'arrière pendant que les nationaux tombaient au combat, s'étaient enrichis immoralement ». Schor, op. cit., note 48, p. 76.
} 
place dans les entreprises françaises. C'est le cas de la section normande, lors d'une assemblée générale au printemps 1934 :

"Ne laissez pas prendre par des étrangers des places qui devraient revenir en premier lieu à des Français (...) dans la région normande opèrent de nombreux chimistes étrangers qui sont loin d'être de véritables spécialistes difficiles à remplacer, mais dont la cohésion et l'esprit de solidarité m'ont beaucoup frappé. Contrairement à ce que pensent beaucoup d'entre vous, si la législation en vigueur permet, grâce à un mécanisme spécial, de s'opposer à l'introduction d'un ingénieur étranger lorsque "l'introduction de ce travailleur porte préjudice aux travailleurs français au chômage", elle ne peut rien contre ceux déjà installés en France, et Dieu sait s'ils sont nombreux, à condition bien entendu que leurs papiers soient en règle. Parmi les différents moyens propres à assurer la sauvegarde des intérêts des ingénieurs français la Section normande, dans sa séance du 25 février dernier, a émis le vœu que les demandes de naturalisation émanant d'ingénieurs étrangers fassent l'objet d'enquêtes sévères et que seules quelques unes soient prises en considération. Selon une formule bien connue, nous demandons là une chose naturelle: "La France aux Français" " 51 .

À aucun moment, les syndicalistes qui s'expriment ne prennent en compte la situation objective du pays. Il est vrai que la France est un pays d'immigration, mais c'est une nation où avant guerre, le nombre de décès pouvait excéder celui des naissances. Ce sera de nouveau le cas à la fin des années trente. La guerre a fait des ravages: la population active industrielle a été amputée de près de $9 \%$. Il faut ajouter l'arrivée sur le marché du travail des classes creuses. En 1931, la population active comptait pour 52,4\% des Français. En 1936, elle

\footnotetext{
51 Discours d'Eugène Simon, président de la section normande. I.C., $\mathrm{n}^{\circ} 134$, mai 1934.
} 
n'était plus que de $49,2 \%^{52}$. Il n'y a pas de la part de l'État une politique raisonnée de l'immigration, elle est laissée aux soins des organismes patronaux. La fonction des pouvoirs publics se réduit à une police des étrangers qui se manifestera par des législations successives dont les deux lois du 11 août $1926^{53}$ et du 10 août $1932^{54}$, cette dernière aboutissant à des expulsions d'étrangers entrés légalement. Manifestement, ces textes qui veulent répondre aux peurs d'une population qui se méfie des étrangers, ne suffisent pas aux chimistes $^{55}$. Mais la crainte de la section normande va au-delà puisqu'elle désigne les naturalisés considérés comme trop nombreux. La loi du 10 août 1927 a facilité la naturalisation des étrangers face à la faiblesse de la natalité française et au manque de main d'œuvre dans de nombreux secteurs. Dans son article 6, cette naturalisation est accordée, entre autres, aux étrangers ayant vécu sur le sol français au moins un an et ayant obtenu des diplômes délivrés par les facultés françaises, ce qui est le cas d'une partie des chimistes formés dans les instituts techniques des facultés des sciences. Or l'application de cette loi a provoqué des mouvements de protestation d'une série de professions qui se traduisent par des législations ad hoc. La loi

\footnotetext{
${ }^{52}$ Chiffres empruntés à R. Schor, op. cit., note 48, p. 29.

${ }^{53}$ La loi introduit l'exigence d'une carte d'identité de «travailleur ». Elle interdit d'embaucher un étranger sans présentation de cette carte qui indique la profession du porteur. Il est également interdit de changer de métier avant un an, pour éviter l'embauche par un autre employeur. Dans les faits, en fonction des régions et de la situation économique locale, cette législation sera plus ou moins appliquée.

${ }^{54}$ La loi crée un contingentement des étrangers employés. Dans les services publics, la proportion ne peut excéder 5\% (article 1). Dans les entreprises privées, des décrets fixent cette proportion «par profession, par industrie, par commerce ou par catégorie professionnelle, pour l'ensemble du territoire ou pour une région » (art.2).

55 «De façon plus générale, tout étranger, quelle que fut sa nationalité, était jugé dangereux ». Schor R., op. cit., note 48, p. 89.
} 
Armbruster du 21 avril 1933 vise à interdire la pratique médicale aux non-Français et exige la possession d'un diplôme français de médecine, les équivalences étant supprimées ${ }^{56}$. La loi du 19 juillet 1934 écarte les avocats naturalisés du Barreau en exigeant 10 ans de stage préalables. Les ingénieurs, comme l'ensemble des corps professionnels veulent suivre ce mouvement en se plaignant, eux aussi, d'être envahis.

Cette grande crainte est étroitement corrélée à une autre inquiétude qui se transforme en obsession au fil des ans: le surnombre. Dès sa création le syndicat est persuadé qu'il se forme trop d'ingénieurs chimistes. Même dans la période des années vingt où le constat est fait qu'il n'y a pas de chômage, que les jeunes diplômés sont recrutés sans grande difficulté, même s'ils sont peu payés, le raisonnement est le suivant : «Si on donnait aux ingénieurs chimistes les postes seuls pour lesquels ils sont qualifiés, il n'est pas douteux qu'il y en aurait des centaines sur le pavé $\rangle^{57}$. En vertu de ce postulat, une pression est effectuée auprès des directeurs des instituts pour qu'ils diminuent la taille des cohortes d'étudiants (sans doute simultanément avec l'Association commune des anciens élèves). Bien entendu, la crise économique n'arrange rien, mais les informations données par le bulletin restent ambiguës à propos du chômage. Ainsi la section marseillaise et coloniale indique que :

\footnotetext{
${ }^{56}$ Du nom du député, le chirurgien Raymond Armbruster. Cette loi est considérée immédiatement comme insuffisante par les étudiants en médecine et le corps médical qui dénoncent « les métèques ». Comme l'indique Bénédicte Vergez, « les médecins ne refusent pas seulement les médecins étrangers restés étrangers; ils refusent les médecins d'origine étrangère ». Vergez Bénédicte, Le monde des médecins au XX $X^{e}$ siècle, Éd. Complexe, Bruxelles, 1996, p. 70. En 1935, la loi sera durcie contre les naturalisés « indésirables ».
}

${ }^{57}$ I.C., n०84, novembre 1929. 
«nos écoles produisent trop d'ingénieurs et ces derniers sont obligés à leur sortie d'école de se déclasser voire d'abandonner la profession. Nous devons élever la voix vers une réduction des effectifs diplomés et même des écoles. Si l'on faisait une statistique judicieuse du nombre des techniciens diplômés qui - d'après certains directeurs d'établissement est nécessaire pour l'existence de leurs écoles (...). Les ingénieurs chimistes voient actuellement leur situation limitée, stagnante, sans amélioration matérielle, même parfois des réductions d'appointements qui - si elles ne sont pas acceptées par le technicien en fonction - sont aussitôt acceptées par le jeune chimiste sans place ${ }^{58}$.

La question est de déterminer quelle serait le nombre optimal d'ingénieurs chimistes à mettre sur le marché du travail chaque année. Un des dirigeants syndicaux, Paul Dubois, s'exprime ainsi : «Un problème domine actuellement tous les autres, celui du surnombre des ingénieurs. En considérant l'indice maximum de la production industrielle à base 100 en 1913, pour le premier trimestre de 1930 (avant la crise), l'indice est à 140. En prenant la production annuelle correspondante des chimistes, on a produit 60 chimistes en 1913 et 600 en $1929 »{ }^{59}$. Mais en 1929 , le syndicat ne constatait pas de chômage... En décembre 1934, au plus fort de la crise économique, donc, le syndicat tente (enfin !) de mener une enquête sur les besoins en ingénieurs chimistes. Il constate que les bases manquent. Un questionnaire adressé aux associations d'anciens n'a ramené que 7 réponses: selon les établissements, les services de placement indiquaient une proportion de 10 à $30 \%$ de non placés par rapport à l'effectif total des promotions. Avec ces données parcellaires et donc fragiles, on calcule que sur la base d'environ 400 diplômés par an

\footnotetext{
${ }^{58}$ I.C., $\mathrm{n}^{\circ} 133$, avril 1934.

${ }^{59}$ I.C., $\mathrm{n}^{\circ} 134$, mai 1934.
} 
avec une moyenne de $20 \%$ de non placés, il y a 320 chimistes utilisés. En réalité, tous les ingénieurs n'utilisent pas systématiquement les services de placement des associations. Beaucoup d'autres réseaux peuvent être mobilisés. L'UIC fournit les résultats d'une enquête ne portant que sur 14 établissements importants - alors que l'essentiel des entreprises chimiques est composé de petites entreprises. Ces résultats sont extrapolés (comment?) à l'ensemble de l'industrie chimique pour aboutir à un besoin de 389 ingénieurs en 1929. L'auteur anonyme du texte syndical estime que pour avoir les besoins de 1934, il faut rabattre ce chiffre à 300 . Un autre calcul est tenté à partir des indices de la production industrielle et l'indice de 1913 est encore une fois fixé à 100 , mais contrairement à l'analyse de mai 1934, le nombre d'ingénieurs produits en 1913 est établi à 150. L'indice de la production industrielle étant de 97 en 1934, compte tenu du développement de la technique, le rédacteur opte pour des besoins annuels de 200 ingénieurs. Il admet cependant que le chiffre est sans doute trop faible, qu'il n'y a pas d'indice spécifique pour les industries chimiques et qu'en outre, nombre de chimistes sont employés dans des industries diverses comme la métallurgie, le caoutchouc, la soie artificielle, etc. ou qu'ils peuvent travailler dans des emplois commerciaux, des emplois techniques officiels, etc. Alors, au doigt mouillé, il arrive par cette voie à un chiffre de 300 ingénieurs nouveaux utilisables par an. Et de conclure: «Ces méthodes de calcul appellent de sérieuses réserves, elles aboutissent toutes à des résultats voisins: les besoins du pays en ingénieurs chimistes sont de l'ordre de 300 par an ${ }^{60}$.

${ }^{60}$ I.C., n 140 , décembre 1934. 
En janvier 1935, L'Ingénieur-Chimiste rend compte d'une statistique tirée du dernier bulletin de la Société de chimie industrielle établie sur les résultats de 13 écoles de chimie (il manque l'Institut de Nancy) pour 1934, soit 283 diplômés (dont 100 étrangers, chiffre approximatif). Le bulletin rappelle les données d'années précédentes : en 1932, il y avait eu 332 diplômés (dont 133 étrangers), en 1931, 331 (dont 93), en 1929, 417 (dont 175). Le journal syndical est donc amené à constater une très sensible diminution même si elle est inégalement répartie selon les établissements. On trouverait les mêmes tendances dans les écoles d'électricité ${ }^{61}$. Même avec la reprise économique en 1936 qui voient les industriels se plaindre du manque de cadres formés, mais aussi les risques de guerre qui sont de plus en plus menaçants et qui justifient une réactivation des industries de défense, l'augmentation des cohortes ne se fera qu'à la marge.

La loi du 10 juillet 1934 qui régule la délivrance des diplômes d'ingénieurs est critiquée parce qu'elle laisse libre l'usage du mot « ingénieur» et les travaux de la commission des titres d'ingénieurs chargée de dresser la liste des établissements habilités à délivrer ce parchemin est blâmée parce que cette liste est trop longue. Cette perception inquiète n'est pas propre aux ingénieurs. C'est un phénomène qui touche l'ensemble de la société dans l'entre-deuxguerres et dont on retrouve l'écho dans les publications de la Confédération des travailleurs intellectuels. Dans de nombreux corps de métiers, on soutient qu'il y a trop de professionnels ou de prétendus

\footnotetext{
${ }^{61}$ C'est notamment le cas des écoles privées parisiennes d'électricité. Voir : Grelon André, «Les origines et le développement des écoles d'électricité Breguet, Charliat, Sudria et Violet avant la Seconde Guerre mondiale », Bulletin d'histoire de l'électricité, 11, 121-143 (juin 1988).
} 
tels, et qu'il faut éliminer ceux-ci et réduire le nombre de ceux-là : par exemple, la loi du 29 mars 1935 établissant une carte de journaliste et confiant la sélection à une commission va dans ce sens. Les professions libérales réclament, elles aussi, une réglementation drastique $^{62}$. Mais, d'une façon générale, les outils statistiques sont trop frustres, quand ils ne manquent tout simplement pas, pour pouvoir appuyer objectivement des revendications qui ne reposent bien souvent que sur l'air du temps.

\section{D'autres revendications}

À côté de ces questions qui taraudent le syndicat et qui occupent la plupart des pages du bulletin, d'autres revendications qui sont clairement de nature syndicale n'occupent qu'une place restreinte, voire sont à peine évoquées une à deux fois durant les vingt ans d'existence de la publication.

Ainsi en est-il de la question des brevets des produits chimiques et pharmaceutiques, thème qui devrait intéresser au premier chef des ingénieurs chimistes. Il est abordé en octobre 1928 à propos d'une nouvelle législation à ce propos qui a fait surgir de sérieuses divergences entre la Chambre et le Sénat. On oppose toujours la doctrine française (brevet de produits) à la doctrine allemande (brevets de procédés), débats qui remontent à Thenard et Gay-Lussac (alors

\footnotetext{
62 Les médecins réclament un ordre dès 1928. Vichy leur offrira un Conseil supérieur des médecins (loi du 7 octobre 1940). L'ordre des architectes est préparé en 1937, par Jean Zay, ministre de l'Instruction publique. Il sera établi par la loi du 31 décembre 1940. Quant aux pharmaciens qui veulent eux aussi un ordre en 1939, l'État de Vichy y répondra par des conseils régionaux et un conseil national. Toutes ces législations seront revues à la Libération, mais le principe des ordres sera confirmé.
} 
que la loi de 1844 comprenait les deux) et qui ont toujours conclu au statu quo. Mais, dit l'auteur de l'article :

"les nouveaux remèdes ne sortent plus de l'officine du pharmacien. Ils ne peuvent être trouvés que dans les laboratoires puissamment outillés, par des chimistes excellents, documentés par des experts bibliographes. Le corps créé doit être étudié expérimentalement par d'habiles cliniciens ; il faut réunir, avant de commercialiser le produit, un ensemble de travaux qui ne peuvent être exécutés que par des maisons disposant de ressources considérables. La fabrication elle-même est des plus difficiles et doit être constamment contrôlée ${ }^{63}$.

Le bulletin revient sur la question le mois suivant en proposant une analyse historique de la découverte de nouveaux produits colorants. Quelle est la responsabilité de la jurisprudence de la loi de 1844 ne reconnaissant que le brevet de produit et considérant comme un perfectionnement le brevet de procédé dans la ruine de l'industrie des matières premières colorantes en France? L'auteur invoque des causes multiples, indique qu'aucun argument sérieux ne justifie l'interdiction de breveter les produits chimiques et pharmaceutiques, mais il ne propose aucune analyse justifiant une prise de position du syndicat, aucun mot d'ordre pour lancer une campagne ${ }^{64}$. On en restera là et la thématique des brevets ne sera plus abordée. Est-ce parce que cela ne concerne pas la majorité des syndiqués?

Le problème des maladies professionnelles est également abordé de façon succincte à propos de la publication d'un rapport présenté à l'Académie de médecine sur le contrôle et la réglementation des établissements industriels qui s'occupent de la

\footnotetext{
${ }^{63}$ I.C., n०72, octobre 1928.

${ }^{64}$ I.C., n ${ }^{\circ} 73$, novembre 1928.
} 
préparation des corps radioactifs. Ce rapport se conclut par un vœu au ministre du Travail et de l'Hygiène qui est résumé ainsi :

"Que les établissements industriels où l'on prépare, manipule ou transporte des corps radio-actifs soient classés parmi les établissements insalubres, non point pour le voisinage, mais au point de vue des travailleurs qu'ils emploient; qu'une réglementation et une surveillance étudiées dans le détail par une commission administrative scientifique et technique soient imposées à ces établissements en ce qui concerne l'hygiène du travail $»^{65}$.

On en restera là, à part la mention en 1939 de l'extension de la liste des maladies professionnelles donnant droit à réparation ${ }^{66}$.

Une protestation véhémente du syndicat est adressée aux pouvoirs publics à propos d'un projet de réglementation des laboratoires d'analyse médicale qui exige le titre de médecin ou de pharmacien pour diriger de tels établissements. «On prétend exclure d'une profession même ceux qui la pratiquent depuis des années puisqu'il semble même que les laboratoires existants devront s'adjoindre un médecin ou un pharmacien ». Le bulletin souligne amèrement que la commission du projet ne comprenait aucun chimiste $^{67}$. Aucune allusion ne sera plus faite à ce problème.

En janvier 1935, le conseil d'administration du syndicat soulève un problème pertinent pour les ingénieurs, à propos de la chute de la maison Citroën en 1934 :

«La défaillance de la société Citroën pose de façon brutale un problème dont les syndicats d'ingénieurs ont eu déjà à s'occuper, mais de façon fragmentaire et généralement

\footnotetext{
${ }^{65}$ I.C., n³6, juin 1925.

${ }^{66}$ I.C., $\mathrm{n}^{\circ} 168$, janvier 1939.

${ }^{67}$ I.C., $\mathrm{n}^{\circ} 146$, juin 1935 et $\mathrm{n}^{\circ} 147$, juillet-août-septembre 1935.
} 
individuelle. Il semble logique de considérer, en effet, que les ingénieurs n'apportent pas seulement leur collaboration technique à l'entreprise dont ils font partie mais qu'ils lui font, en outre, un apport créateur et de perfectionnement dont il n'est tenu nullement compte quand il s'agit de rompre le contrat qui les lie avec l'entreprise défaillante $»{ }^{68}$.

Le C.A. plaide pour une campagne énergique qui serait le point de départ d'une véritable politique syndicale. On voyait là l'amorce d'une réflexion sur la responsabilité des cadres au sein des entreprises et le rôle qu'ils pourraient jouer dans la gestion de ces établissements. Ces questions seront reprises par le syndicalisme cadre après la Seconde guerre mondiale. Mais une fois encore, le syndicat des chimistes ne va pas au-delà de cette seule mention.

\section{Le problème des ingénieurs âgés}

La question des ingénieurs âgés et des difficultés qu'ils pourraient rencontrer sur le plan professionnel est mentionnée une première fois dès 1921 à propos de la question des retraites. La législation sur les retraites ouvrières et paysannes par les lois du 5 avril 1910 et du 27 février 1912 rendait l'assurance obligatoire pour tous les salariés dont le salaire ne dépassait pas $3000 \mathrm{f} / \mathrm{an}^{69}$. Ce montant avait été réévalué à 5000 f/an par la loi du 20 décembre 1918. Le rapport présenté à la commission du statut indique que le minimum d'appointement pour les ingénieurs chimistes étant de 9000 f/an, les avantages de ces lois ne peuvent leur être appliqués. Selon ce rapport, c'est au patron qu'incombe le paiement des primes pour l'assurance

\footnotetext{
${ }^{68}$ I.C. $\mathrm{n}^{\circ} 142$, février 1935 .

${ }^{69}$ Il s'agissait de retraite par capitalisation, prélevée sur le salaire.
} 
d'une retraite à son ingénieur. Les versements devraient être effectués à la Caisse nationale des retraites pour la vieillesse qui fonctionne sous la garantie de l'État. Si la revendication est précise, elle ne sera pas renouvelée, faute d'interlocuteur. Même la création des assurances sociales en 1930 n'apportera aucune satisfaction aux ingénieurs et cadres $^{70}$.

Une deuxième mention relative aux ingénieurs âgés se situe en 1929, au moment de la célébration des dix ans du syndicat. Le rapport moral de l'assemblée générale est présenté par le président Landowski qui signale la difficulté pour trouver des places pour les ingénieurs chimistes d'un certain âge, car c'est une tendance générale de l'industrie que de rajeunir les cadres :

"et c'est malheureusement et trop souvent sans ménagements que sont remerciés des hommes ayant 15, 20 ans et plus de séjour dans une maison. Il y a là une source de grave préoccupation pour notre syndicat, comme pour les autres syndicats d'ingénieurs et malheureusement, en ce moment, il n'y a pas de remède décisif à cette situation ${ }^{71}{ }^{7}$.

Un peu plus tard, le service de placement du syndicat indique que :

" [Les industriels] ne demandent pas et même ils ne veulent pas d'ingénieurs d'un certain âge, même avec de bonnes références, même ayant exercé des fonctions importantes. On ne recrute pas pour les postes supérieurs. Il y a là une situation de fait que

\footnotetext{
${ }^{70}$ La loi du 30 avril 1930 sur les assurances sociales avec une section retraite obligatoire par capitalisation ne s'applique que pour les salariés en dessous d'un plafond. Les ingénieurs doivent donc se constituer une retraite par eux-mêmes. Il faudra attendre les ordonnances des 5 et 9 octobre 1945 pour voir créé un régime général de sécurité sociale. Il s'applique obligatoirement à tous les salariés du secteur privé, quel que soit le montant de leur salaire. La retraite s'effectue par répartition. Le système sera complété en 1947 pour les cadres avec la création de l'AGIRC qui gère leurs retraites complémentaires.

${ }^{71}$ Compte rendu du rapport moral de l'AG du 24 février 1929. I.C., $\mathrm{n}^{\circ} 77$, mars 1929.
} 
nous déplorons, contre laquelle nous nous élevons, qui est d'une extrême gravité sociale, qui demandera une intervention législative mais que le service de placement ne peut que subir ${ }^{72}$.

Le problème des vieux chimistes n'est plus abordé jusqu'en fin 1935. Le conseil d'administration du 2 décembre 1935 mentionne que des lettres reçues au siège posent de façon nette la question des chimistes âgés «qui ne peuvent plus ni trouver d'emploi ni souvent réussir à subsister de façon précaire par leurs propres moyens ». Un appel sera fait à la solidarité des groupements de chimistes et d'organisations patronales pour créer un organisme qui recueillerait des fonds soit pour entretenir des places dans des maisons de retraite ou pour des emplois permettant d'améliorer la situation des vieux chimistes ${ }^{73}$.

Un long article-témoignage est publié en mai 1936 sous la signature de J. Boisseau, ingénieur chimiste de l'Institut de chimie de Paris, sous la rubrique «Fins de carrière ${ }^{74}$. C'est un bilan, car les diplômés des premières promotions d'ingénieurs chimistes ont atteint la soixantaine. L'auteur reconnaît que certains ont des fins de carrière heureuses et il rend hommage aux établissements «qui ont prévu la retraite de leurs fidèles collaborateurs dans l'organisation de caisses qui leur assurent toute sécurité pour l'avenir ». Mais ce n'est pas le plus grand nombre.

«Nos associations, notre syndicat ont eu la preuve douloureuse qu'un ingénieur chimiste dont la vie n'a connu que l'équilibre, le travail, l'ordre et le dévouement, pouvait terminer son

\footnotetext{
${ }^{72}$ I.C., nº79, mai 1929.

${ }^{73}$ I.C., $\mathrm{n}^{\circ} 151$, janvier 1936.

${ }^{74}$ I.C., $\mathrm{n}^{\circ} 155$, mai 1936.
} 
existence dans un dénuement, une solitude, un abandon indignes d'une fonction aussi respectable que la nôtre! ».

Le texte indique qu'à la sortie de l'école, même en période de dépression économique, on peut trouver un emploi relativement facilement. Jeunes gens non mariés, sans charge, «ils peuvent accepter des conditions d'émoluments et d'habitat les plus variées ». Ensuite, pour la plupart d'entre eux, la crise augmente de façon considérable les risques d'insécurité : diminution des frais généraux, dépôt de bilan, voire arrêt complet, on peut se trouver brusquement sans situation. Quand on n'est plus jeune chimiste, les difficultés commencent. «Au début de la crise, les services de placement savaient qu'il était très difficile de trouver une situation aux plus de 45 ans. Depuis, la limite d'âge s'est terriblement abaissée » alors qu'un chimiste au métier essentiellement expérimental devrait être au contraire apprécié pour ses années d'expériences.

"Les directions d'industrie, les conseils d'administration, les postes de tous ceux qui sont des dirigeants d'affaires sont-ils confiés uniquement à des jeunes? Pourquoi les qualités exigées pour ceux qui doivent être les animateurs d'une activité industrielle deviennent-elles sans utilité quand il s'agit de leurs collaborateurs éventuels? ». La tentation serait donc de changer d'orientation, mais on ne peut pas lui demander d'avoir des qualités universelles. Alors, «il va sentir l'amertume d'avoir travaillé pendant sa jeunesse pour l'obtention d'un diplôme qui lui apparaît de plus en plus comme une duperie ».

Depuis longtemps, conclut l'ingénieur, le syndicat se préoccupe de ce problème. On a lancé l'idée généreuse d'une Maison du Chimiste, irréalisable pour l'heure. On cherche à créer un fonds national d'aide aux ingénieurs chimistes âgés, un comité d'entraide est en voie de constitution. 
S'il n'est pas douteux, en lisant les témoignages, que des ingénieurs se sont retrouvés au chômage au moment de la crise économique, il est impossible de déterminer l'ampleur du phénomène : les ingénieurs en tant que tels ne sont pas comptabilisés dans la statistique publique dans l'entre-deux-guerres. On l'a vu plus haut, le syndicat des chimistes, sur la base de ses propres enquêtes, est dans l'incapacité de produire des chiffres fiables, étant donné la faiblesse des données recueillies. Cela touche-t-il plus particulièrement les ingénieurs âgés ? La notion d'âge, telle qu'elle est utilisée dans les différents textes qui abordent cette question, est floue, ou plus exactement elle est particulièrement large, puisqu'un des exemples fournis parle d'un ingénieur de 32 ans, déjà jugé trop vieux, cas limite sans doute ${ }^{75}$. À l'autre extrémité du continuum, le bulletin abordera la question des ingénieurs retraités nécessiteux. Mais, en fait, l'accent est mis sur les ingénieurs qui, ayant déjà une carrière riche et des qualifications indéniables, ayant rendu de signalés services aux entreprises qui les ont employés, se retrouvent sur le marché du travail et à qui aucune offre ne serait proposée. C'est le caractère profondément injuste de la situation subie par ces experts expérimentés qui suscite l'émotion et l'indignation.

Cette question n'est pas propre aux ingénieurs chimistes, même si les exemples apportés dans le bulletin ne traitent que de leur seul domaine. On retrouve les mêmes cas, jugés tout aussi scandaleux, et dénoncés de la même façon chez les ingénieurs électriciens. En réalité, l'USIF qui regroupe les syndicats de chimistes, d'électriciens et de mécaniciens a fait du problème des ingénieurs âgés un des fers

\footnotetext{
${ }^{75}$ Ibid.
} 
de lance de son action syndicale. Petite organisation minoritaire d'un groupe professionnel étroit, compartimenté, qui peine à faire reconnaître une existence autonome face à la masse ouvrière organisée et à la puissance patronale, elle a construit un argumentaire autour d'une figure emblématique et consensuelle qui peut toucher le monde social, bien au-delà du corps des ingénieurs ${ }^{76}$.

"[Cette image] rapproche le groupe professionnel au plus près de la société qui a élu la gestion de la vieillesse comme un grand problème social et économique au tournant de ce siècle $»^{77}$.

Lancé par l’USIF, le « scandale » des ingénieurs âgés est repris par les autres syndicats d'ingénieurs et par la fédération des associations d'anciens élèves qui en font une cause nationale. La grande presse s'en empare qui trouve là un sujet porteur. Mais à aucun moment, quelle que soit la publication, des données quantitatives viennent étayer la démonstration. Et les travaux des historiens qui portent sur la gestion du personnel dans les entreprises durant la période montrent plutôt la recherche d'une stabilité et une fidélisation des employés qui permettent de s'appuyer sur des compétences reconnues ${ }^{78}$. Rien en tout cas qui puisse justifier l'hypothèse d'un évincement massif des ingénieurs âgés.

\footnotetext{
${ }^{76}$ « Moins qu'une revendication sur la "misère" de la crise (...), le chômage du vieil ingénieur devient un référent symbolique. Il se transforme en allégorie du malheur, en représentation emblématique par l'ampleur de la crise, à partir de laquelle 1'"ingénieur âgé" parvient à incarner l'homme tragique sur qui les souffrances s'accumulent. Cette figure imaginaire condense en elle-même les événements les plus douloureux, de sorte que l'histoire révèle l'indignation, une injustice qui doit cesser ». Hugot-Piron Nathalie, Les "cadres âgés ». Histoire d'une catégorie de chômeurs, Presses universitaires de Rennes, Rennes, 2014, p. 93.

${ }^{77}$ Ibid.

${ }^{78}$ Id., p.103-105.
} 


\section{Repenser la formation}

La question de la formation obsède le syndicat dès son origine. On se souvient que parmi les toutes premières revendications émises, une des plus détaillées concernait l'enseignement de la chimie industrielle qu'il fallait régénérer, terme fort qui définissait bien le peu d'estime dans laquelle les chimistes, pourtant issus des établissements délivrant cette formation, la tenait. Une des raisons était que les instituts, créés au sein des facultés des sciences, n'avaient pas été conçus selon une logique centralisée ${ }^{79}$ : les plans d'études n'étaient pas homogènes, les modes de recrutement étaient organisés en fonction du bassin régional d'éducation et les ingénieurs estimaient qu'il manquait des enseignements pratiques, c'est pourquoi ils préconisaient que des cours fussent donnés par des techniciens qualifiés. Et pour être sûrs du suivi de ces réformes, ils demandaient que des ingénieurs, issus préférentiellement de leur institut, soient membres de son conseil de perfectionnement.

Mais les raisons n'en étaient pas uniquement scientifiques. Les chimistes souhaitaient que le diplôme d'ingénieur chimiste « requalifié » soit reconnu par l'État et donne accès, au même titre que les écoles d'application de Polytechnique, à tous les postes de l'État utilisant des chimistes. De même, se souvenant des conditions de leur

\footnotetext{
79 «Pour arriver à se défendre, les ingénieurs-chimistes doivent former un bloc intellectuel, c'est-à-dire que le titre d'Ingénieur-chimiste doit correspondre à quelque chose de bien défini. Or, à l'heure actuelle, les différentes écoles de chimie qui forment des ingénieurs-chimistes ont des programmes différents qui confèrent à leurs élèves une formation chimique et surtout une formation générale variable. L'ingénieur chimiste ne peut par suite se définir nettement ». I.C., $\mathrm{n}^{\circ} 11$, octobre 1920.
} 
affectation militaire au moment de la guerre, ils exigeaient un statut militaire spécial - sans nul doute pour ne pas se retrouver sous la coupe des ingénieurs des Poudres. Enfin, il ne faut pas oublier la conception singulièrement malthusienne du monde des ingénieurs qui s'imaginaient toujours trop nombreux ${ }^{80}$ : en montant singulièrement le niveau de formation, on diminuerait le nombre de diplômés.

La question du diplôme prend, dans l'entre-deux-guerres, une dimension qu'elle n'avait jamais connue antérieurement. $\mathrm{Au} \mathrm{XIX}$ siècle, le Conservatoire des arts et métiers dont les enseignements étaient donnés par des professeurs prestigieux, avec des programmes s'étalant sur plusieurs années, devant des centaines d'auditeurs, ne délivrait aucun diplôme. Il en était de même au Muséum d'histoire naturelle et dans les cours du soir offerts par des structures bénévoles comme l'Association polytechnique ou l'Association philotechnique. Les cours publics des facultés des lettres et des sciences qui étaient assurés par les professeurs titulaires étaient essentiellement destinés à un public cultivé plus qu'aux quelques étudiants de licence. L’École polytechnique elle-même n'attribuait pas de diplôme et le titre d'ingénieur obtenu à l'issue de la formation dans les écoles d'application constituait un grade dans une hiérarchie professionnelle. La création des universités en 1896 et les décrets de 1897 les autorisant à ouvrir des cours à d'autres fins que l'obtention des grades d'État, pour délivrer des diplômes d'université, ont modifié la donne. Les instituts annexes des facultés des sciences qui se créent alors obtiennent par les conseils d'université de créer des diplômes

\footnotetext{
${ }^{80}$ Vœux de la section parisienne dans sa réunion du 26 juin 1920 : «Limiter le nombre des entrants dans les écoles de chimie afin d'éviter une surabondance d'ingénieurs dans quelques années ». I.C. n8-9, juillet-août 1920.
} 
d'ingénieurs, et ces décisions sont systématiquement avalisées par la direction des enseignements supérieurs. Les écoles d'arts et métiers décrochent à leur tour le droit de délivrer, à partir de 1907, un brevet d'ingénieur et les écoles privées d'électricité qui disent vouloir produire « les gadzarts de l'électricité » décident alors de sanctionner leurs formations par un diplôme d'ingénieur. Il reste malgré tout beaucoup de «non diplômés » désignés, souvent à tort, comme autodidactes. Dans la catégorie des « ingénieurs », ils seraient à peu près autant que les diplômés estimés à 60000 entre les deux guerres. Quand il se constitue en 1919, le SICF dresse la liste des « chimistes qualifiés » qui ont vocation à adhérer et après avoir listé les diplômés des instituts de chimie, il accepte en deuxième rang les «élèves de la première catégorie ayant accompli toute leur scolarité ${ }^{81} \ldots$ mais qui n'ont pas de diplôme, et il étend son recrutement en fin de compte à ceux qui n'entrent dans aucun des établissements listés, mais qui peuvent justifier d'une longue pratique de chimiste avec des responsabilités effectives, car, on l'a vu plus haut, dans le monde de l'entreprise, le diplôme est encore peu ou pas valorisé.

Toutefois, phénomène bien connu en sociologie des professions, à partir du moment où un groupe professionnel tient à se constituer en tant que tel, il s'agit de conquérir le droit de pratiquer son métier jusqu'à obtenir le monopole de son exercice. Dans cette lutte des places, le diplôme institue une distinction entre les empiristes qui ont acquis des savoir-faire sur le tas et ceux qui, grâce à leurs études, disposent d'un savoir théorique qui encadre, explicite, donne du sens à la pratique. Le diplôme légitime les seconds et tend à

\footnotetext{
${ }^{81}$ Voir annexe 1.
} 
disqualifier les premiers. Encore faut-il que le diplôme lui-même apparaisse comme suffisamment établi pour fonder cette légitimité. C'est tout l'enjeu de la bataille que doit mener le syndicat qui se veut le représentant authentique de l'ensemble des chimistes professionnels. D'un côté, il faut disqualifier les établissements ou les filières dont le niveau d'enseignement ne correspondrait pas à ce qui serait requis pour être chimiste «qualifié ». De l'autre, il faut militer pour arracher aux instituts universitaires, jaloux de leurs prérogatives, une harmonisation visant à faire des diplômes d'ingénieurs chimistes un diplôme supérieur qui le mettrait à équivalence fonctionnelle des diplômes des grandes écoles, avec la reconnaissance du parchemin par l'État.

Rapidement, une attaque violente est menée contre l'École universelle, une structure privée de formation par correspondance, créée en 1907 et qui connaît alors un vif succès. Elle délivre un «brevet d'études générales et techniques relatives à la profession d'ingénieur-chimiste». Elle est dénoncée comme «chimie sans laboratoire ni hydrogène sulfuré », c'est-à-dire sans formation à la paillasse, et l'auteur défie quiconque «de former un analyste même médiocre à l'aide de bouquins ». La conclusion s'impose d'ellemême : "Pour nous, ingénieurs chimistes, nous considérons comme inexistants les titres donnés par cette école soi-disant universelle ${ }^{82}$. La cause paraît entendue, et pourtant, cette position soulève des interrogations et des débats à l'intérieur du syndicat. Des témoignages indiquent avoir vu des employés travailler d'arrache-pied pendant plusieurs années : "Ils sont arrivés à posséder une excellente culture

${ }^{82}$ I.C., $\mathrm{n}^{\circ} 4$, mars 1920. 
scientifique qui peut très largement être comparée à celle qu'on acquiert dans une école et sur place», dit l'un. Un autre correspondant conclut :

«Il n'est donc pas exact de continuer à déclarer que ces écoles délivrent des diplômes à la légère, à des candidats indignes du titre. Je considère même que ces écoles rendent des services inestimables en améliorant énormément des jeunes gens pauvres mais bien doués, souvent guidés dans leurs études par leurs chefs d'entreprises et qui accroissent leur valeur personnelle sans abandonner la situation qui les fait vivre ${ }^{83}$.

Le mois suivant, le bulletin fait état d'une correspondance nombreuse avec beaucoup d'approbations, mais aussi majoritairement des réponses défavorables. La direction clôt le débat de façon assez abrupte :

«On peut apprendre l'ensemble des lois et fait relatifs à la chimie théorique. Mais ce n'est pas l'essentiel de l'art d'un chimiste: l'important est un certain métier manuel et surtout l'habitude de l'observation et de l'expérimentation. Dans les instituts de chimie, une place capitale est faite au laboratoire. Ce n'est pas possible par correspondance ».

Ceux qui se bornent à un enseignement théorique ne seront jamais ingénieurs chimistes. Un diplôme par correspondance ne peut accorder aucune connaissance pratique et donc droit à l'admission. Son possesseur devra justifier d'un stage professionnel ${ }^{84}$.

Ainsi se définissent les caractéristiques professionnelles du chimiste: outre une formation théorique, l'observation et l'expérimentation sont indispensables. Cette position qui permet de rejeter les techniciens formés par correspondance est également

\footnotetext{
${ }^{83}$ I.C., $\mathrm{n}^{\circ} 70$, juillet 1928 .

${ }^{84}$ I.C., n71, août-septembre 1928.
} 
opposée à l'analyse d'Henry Le Chatelier. Le savant, connu comme un redoutable polémiste, émet, dans un article de Chimie et industrie de mai 1920, un point de vue tranché qui peut se résumer, selon Émile Fleurent qui tient à lui répondre, en trois points : 1 . Pour être chimiste, l'essentiel est d'avoir une forte culture mathématique; 2. Le chimiste dans ses travaux doit se laisser guider par des lois mathématiques d'une certitude absolue, et contre lesquelles ne peuvent prévaloir les faits expérimentaux ; 3. Il n'y a pas d'écoles de chimie capables de former des chefs de laboratoire. Quant aux chefs de fabrication, directeurs, chefs d'industrie, ils ne peuvent être recrutés que parmi les élèves à formation «polytechnique». Évidemment, le président du SICF pose que toute la chimie organique et une large partie de la chimie minérale «restent encore à présent en dehors de la spéculation mathématique ». Il dénonce la hiérarchie industrielle validée par Le Chatelier dans laquelle «les sommets sont fournis par les élèves des grandes écoles techniques, le dernier échelon par les manœuvres et l'avant-dernier par les chimistes et physiciens "expérimentaux" ». Le Chatelier ayant indiqué que l’École des mines constituait selon lui l'école supérieure de chimie dont la France avait besoin, Fleurent affirme que cet établissement n'est pas une école de chimie, car la chimie qu'on y enseigne est bien trop spécialisée : «Pour prétendre au titre de chimiste, il faut savoir autre chose que la métallurgie $»^{85}$.

Que faire alors? Il faut obtenir l'unification de l'enseignement dans les écoles de chimie. L'Union des associations d'anciens élèves en a conçu le programme dans son congrès de mars 1920 ; il suffit de

${ }^{85}$ I.C., ${ }^{\circ} 72$, octobre 1920. 
s'y référer. Il faut obtenir un diplôme d'État avec tous les avantages qui lui sont attachés. Ce double résultat doit être inscrit dans une loi identique à celle qui a organisé l'enseignement agricole en 1918 et qui protège les titres d'ingénieur agronome et d'ingénieur agricole ${ }^{86}$. Les ingénieurs chimistes peuvent faire valoir que les instituts dont ils sortent relèvent de l'enseignement public et que ce que le ministère de l'Agriculture a pu faire pour les ingénieurs relevant de son secteur, le ministre de l'Instruction publique doit être en mesure d'en faire autant.

C'est devant l'Assemblée générale du syndicat, le 25 janvier 1925, que René Sordes présente un vaste exposé sur le diplôme d'État d'ingénieur chimiste. Il rappelle la création d'une commission mixte du syndicat avec des représentants de l'UNADEC le 24 juin 1924, qui a abouti à la rédaction d'un projet de $10 i^{87}$.

«Pour que le projet se réalise, il faut, outre la sympathie du législateur et des pouvoirs publics, que chacun de nous éloigne

\footnotetext{
${ }^{86}$ L'article 7 de la loi du 2 août 1918 sur l'organisation de l'enseignement agricole attribue respectivement les titres d'ingénieur agronome et ingénieur agricole aux anciens élèves de l'Institut national agronomique et des écoles nationales d'agriculture. " Ceci leur offre donc une protection juridique pour toute usurpation de leurs titres, un atout considérable si l'on considère que les ingénieurs diplômés d'autres établissements d'enseignement technique vont devoir militer jusqu'en 1934 pour une loi protégeant leurs titres. À la différence d'autres titres d'ingénieurs diplômés, qui doivent être suivis du sigle de l'école correspondante, ceux d'ingénieur agricole ou d'ingénieur agronome signalent à eux seuls une appartenance institutionnelle et une formation précise. De même, la définition d'un ingénieur agronome se réduit à sa seule formation: un ingénieur agronome est quelqu'un qui sort de l'Institut national agronomique (INA). C'est leur formation commune, donc, et non pas une activité quelconque professionnelle, qui va permettre aux ingénieurs agronomes de construire une identité collective fondée sur leur titre ». Bénédict-Trocmé Marie, "Le titre d'ingénieur agronome » in Boulet Michel (dir.), Les enjeux de la formation des acteurs de l'agriculture, 1760-1945. Actes du colloque ENESAD, 19-21 janvier 1999, Educagri Éditions, Dijon, 2000, p. 367.
}

${ }^{87}$ Voir annexe 2. 
ce que l'on nomme l'esprit d'école et ne pense, quelque délicat que celui-ci puisse être, qu'au bien général $\gg{ }^{88}$.

L'orateur brosse alors un large rappel historique sur l'enseignement de la chimie depuis l'époque du Jardin du Roy en le situant dans le développement de l'enseignement supérieur ${ }^{89}$. Dans les différentes branches, qu'il s'agisse du développement industriel, de l'agronomie, des facultés de médecine ou de pharmacie,

«tous les organismes créés pour des besoins étudiés et définis ont une discipline d'enseignement, sanctionnée par des diplômes de valeur incontestable et uniforme, fournissant des techniciens parfaitement appropriés aux services qu'on attend d'eux $\|^{90}$.

Il oppose cette organisation à la chimie, où il y a 50 ans, l'industrie sommeillait, l'enseignement se faisait sans laboratoire dans les facultés des sciences, avec quelques rares laboratoires privés qui accueillaient une poignée d'élèves. Aujourd'hui, la chimie n'est plus indépendante des autres sciences, chimie et physique se rejoignent, les mathématiques sont indispensables, le chimiste est tenu d'être au courant de tous les progrès réalisés dans l'ordre technique ou scientifique. Les lois et décrets de 1896 ont modifié le régime des facultés et permis la naissance d'organismes annexes, les instituts de chimie appliquée, qui se sont multipliés et sont au nombre de 14, avec quelquefois plus le souci du nombre que de la qualité des élèves.

\footnotetext{
${ }^{88}$ I.C., n³3, mars 1925 .

${ }^{89}$ Sordes en fera un ouvrage paru en 1928, préfacé par Georges Urbain, lequel décrit l'état désastreux des laboratoires d'enseignement et les ressources misérables affectées aux écoles. Sordes René, Histoire de l'enseignement de la chimie en France. Chimie et industrie, Paris, 1928. La Revue d'histoire de la pharmacie $(R H P)$ qui en fait un compte rendu, qualifie le livre de « petit travail de vulgarisation fort réussi ». Cf. RHP, 19/72 (1931), p. 39-40.
}

${ }^{90}$ Ibid. 
«Contrairement à ce qui a été fait pour les sciences à base mathématique, l'État a laissé se développer au hasard des initiatives, l'enseignement de la chimie appliquée et la formation des ingénieurs-chimistes ${ }^{91}$.

Sordes préconise une formation en deux étapes: d'abord un enseignement scientifique général dans le cadre des facultés avec les certificats de chimie générale, chimie physique, mathématique générale, le certificat de mathématique-physique-chimie (MPC), bagage sanctionné par la licence. Puis un second cycle d'enseignement dans les instituts techniques: chimie appliquée au laboratoire et à l'industrie, physique et mécanique industrielle, technologie, sanctionné par un diplôme d'Ingénieur-Chimiste d'État (DPLG).

Ce diplôme doit être délivré par un jury d'examen unique, accessible uniquement aux Français des deux sexes, non dépendant des facultés ou écoles, composé de représentants qualifiés des enseignants, des industriels et des diplômés. Il comportera trois épreuves, chacune étant éliminatoire. Le diplôme d'État offrira les garanties impossibles à obtenir actuellement de l'ensemble des facultés.

"Les instituts aux moyens trop modestes se contenteront de délivrer leur actuel diplôme. Les écoles délivrant un diplôme correspondant déjà au diplôme qu'on veut instituer, feront bénéficier leurs élèves d'un diplôme apprécié sans risque de confusion $\|^{92}$.

\footnotetext{
${ }^{91}$ Id.

${ }^{92} I d$. En parlant « des écoles », René Sordes pense en réalité plus spécialement à l'École de physique et de chimie industrielle dont le plan d'études correspond au modèle invoqué et qui a donné au pays des ingénieurs et industriels distingués. Cet établissement exerce du reste un discret magistère sur l'USIF dont le fondateur et animateur est Paul Boucherot, un ancien de cette école. Les présidents du SICF qui
} 
L'orateur termine en expliquant que le projet répond à une nécessité immédiate :

«L'État ne peut rester indifférent devant le prodigieux développement de l'industrie chimique, et se doit d'apporter à l'organisation de l'enseignement qui formera les chefs, la même méthode, le même souci de l'effort coordonné qui a présidé à la création des grandes Écoles dont l'enseignement est à base mathématique ${ }^{93}$.

L’UIC, qui a évidemment été avertie de ces débats, décide d'appuyer la démarche du syndicat. Son président en a informé le ministre de l'Instruction publique par une lettre du 21 décembre 1927. Et l'Union nationale des étudiants, dans son congrès de Strasbourg, a émis un vœu favorable à la création d'un diplôme d'État d'ingénieur chimiste. Entrée en contact avec le SICF, elle se propose de s'associer aux démarches auprès du ministère ${ }^{94}$.

En février 1928, le syndicat tient son congrès. Sordes revient sur l'affaire en cours. Il fait état de difficultés. Après l'élan unanime initial, des critiques sont venues : on craint une baisse du niveau des études, on s'inquiète de la contrainte sur les écoles et du risque de disparition de certaines d'entre elles. Victor Grignard lui-même a émis un certain nombre d'objections. L'orateur répond point par point à toutes les remarques. Le plus gênant est que les premières démarches auprès du ministère puis auprès de l'Enseignement technique, sans doute plus qualifié, n’ont jusqu'alors rien donné : "Ces doubles

se succèdent en sont également tous sortis et Sordes lui-même, industriel implanté à Suresnes, a été formé à l'EPCI.

${ }^{93}$ Id.

${ }^{94}$ I.C., n ${ }^{\circ} 64$, janvier 1928. 
démarches ont abouti au classement de fait de notre rapport $\gg{ }^{95}$. On a même senti une opposition. Fleurent, devenu président d'honneur, intervient pour rappeler la démarche de l'UIC auprès du ministre : «La réponse ne vient pas vite! Nous n'admettrons pas que notre projet soit mis sous le boisseau ». Un camarade avait proposé que le syndicat patronal et le SICF organisent eux-mêmes le diplôme. Fleurent a répondu qu'il fallait d'abord tenter d'obtenir satisfaction par le gouvernement. Mais, conclut-il sous les applaudissements, «si nous rencontrons devant nous une résistance absolue, nous reprendrons ce projet ». Le congrès se conclut par une motion précisant le titre du diplôme : diplôme d'ingénieur des arts chimiques, et donnant mandat au Conseil d'administration pour poursuivre encore plus énergiquement la démarche vers la création de ce diplôme d'État.

Un peu plus tard, on apprend qu'une commission a été nommée par le ministre pour étudier la réforme de l'enseignement de la chimie, mais ni l'UIC ni le syndicat n'ont été convoqués ${ }^{96}$. Finalement, Fleurent a pu exposer devant cette commission présidée par Cavalier, directeur de l'enseignement supérieur. À la suite de cette rencontre, un groupe de travail composé de Fleurent, de Petit, doyen de la faculté des sciences de Nancy et directeur de l'école de brasserie, et du professeur de chimie physique de l'Institut chimique de Nancy, prépare un programme complémentaire d'enseignement de physique générale et industrielle, de mécanique pure et appliquée et d'un

\footnotetext{
${ }^{95}$ I.C., $\mathrm{n}^{\circ} 66$, mars 1928.

${ }^{96}$ I.C., $\mathrm{n}^{\circ} 68$, mai 1928.
} 
ensemble de connaissances mathématiques, programme qui est remis à Cavalier ${ }^{97}$.

Le temps passe et les choses n'avancent pas. À l'assemblée générale du 17 février 1934, le président Florentin expose que le Conseil national économique (auquel l'USIF participe) avait fait un vœu l'année précédente en faveur du diplôme d'État d'ingénieur chimiste, transmis officiellement par le président du Conseil, président de droit du CNE, au ministre de l'Éducation nationale : au bout d'un an, il n'y a toujours pas d'accusé de réception. La commission d'enseignement de la chimie est de fait mise en sommeil car elle n'est pas convoquée par la direction de l'Enseignement supérieur. De son côté Georges Urbain a milité pour la création d'une école supérieure de chimie, projet qui serait envisagé avec sympathie par l'Éducation nationale. Le syndicat s'oppose à cette «pure spéculation» qui entraînerait à la mort toutes les écoles de chimie existantes ${ }^{98}$.

Toutefois, un peu plus tard, un contact plus positif a lieu avec le nouveau directeur de l'Enseignement technique, Hyppolite Luc. Une délégation du SICF et de l'UIC apprend au cours de cette rencontre que le projet d'école supérieure est finalement abandonné. Le principe d'un diplôme d'ingénieur chimiste est chaleureusement accepté, sa création est à l'étude au sous-secrétariat à l'Enseignement technique. Une commission sera réunie à la rentrée pour établir les modalités de ce diplôme qui pourra être institué dans le cadre de la loi relative à la protection du titre d'ingénieur, en discussion au

\footnotetext{
${ }^{97}$ I.C., $\mathrm{n}^{\circ} 74$, décembre 1928.

${ }^{98}$ I.C., n ${ }^{\circ} 131$, février 1934.
} 
Parlement ${ }^{99}$. À l'occasion d'une nouvelle rencontre avec le directeur des enseignements, le 3 octobre 1934, Hyppolite Luc laisse entendre que cette question sera remise à l'étude dès que la commission du titre sera nommée et commencera à étudier les questions de son ressort ${ }^{100}$. Mais rien dans l'article du texte de loi du 10 juillet 1934 instituant la commission des titres ne prévoit qu'elle doive se pencher sur la création de diplômes d'État: sa fonction consiste à établir la liste totale des écoles habilitées à délivrer un titre d'ingénieur diplômé, dont les écoles publiques et privées reconnues par l'État, mais elle ne peut statuer que sur l'habilitation des écoles privées non reconnues par l'État (par exemple des écoles catholiques comme l'Institut catholique des arts et métiers, ICAM). Ou Luc méconnaît cette législation, ce qui serait étonnant, ou il induit en erreur des représentants syndicaux trop naïfs. De fait, le CA du 10 décembre 1934 rendant compte des premiers travaux de la commission des titres d'ingénieurs, dont le vice-président est Paul Boucherot, le président de l'USIF, indique qu'elle a dressé la liste des établissements habilités ${ }^{101}$. En définitive, le rapport moral de l'Assemblée générale du 9 mars 1935 indique que la loi sur le titre d'ingénieur ne peut être utilisée pour mettre en œuvre le diplôme d'État ${ }^{102}$. Il ne sera plus jamais fait mention de rencontre avec la direction de l'Enseignement technique. Une dernière allusion à l'éventuelle création du diplôme d'État est faite au cours du CA du 3

\footnotetext{
${ }^{99}$ I.C., n¹37, juillet-août-septembre 1934.

${ }^{100}$ I.C., $\mathrm{n}^{\circ} 139$, novembre $1934, \mathrm{CA}$ du $1^{\mathrm{er}}$ octobre et $\mathrm{n}^{\circ} 140$, décembre $1934, \mathrm{CA} \mathrm{du}$ 5 novembre.

${ }^{101}$ I.C., $\mathrm{n}^{\circ} 141$, janvier 1935.

${ }^{102}$ I.C., n ${ }^{\circ} 142$, février 1935 , l'assemblée étant le 9 mars, le rapport est donc présenté à l'avance.
} 
juin 1935 qui commente les travaux de la commission des titres en indiquant que celle-ci semble fonctionner comme un organisme de maintien des diplômes existants plus que comme une commission de triage des titres en appréciant leur valeur relative et qui conclut que «la seule solution sera le diplôme d'État ${ }^{103}$.

À part le moment où le chimiste Paul Petit, directeur de l'École de brasserie de Nancy, a été sollicité pour élaborer une partie de la formation du futur diplôme d'État, on ne voit pas le syndicat s'intéresser aux établissements de formation autrement que pour des déclarations lapidaires sur le nombre trop élevé d'élèves, le laxisme supposé des directeurs et le niveau a priori médiocre de la formation, mais rien sur leurs difficultés matérielles ${ }^{104}$, sur les travaux des laboratoires, sur la mise en œuvre des diplômes d'ingénieursdocteurs ${ }^{105} \ldots$ Les instituts sont seuls face à leurs problèmes quotidiens. L'attitude du doyen de Nancy montre pourtant qu'il n'y avait pas forcément d'opposition à la mise en œuvre d'un diplôme d'État. Mais quand ces perspectives s'évanouissent, les établissements

\footnotetext{
${ }^{103}$ I.C., $\mathrm{n}^{\circ} 147$, juillet-août-septembre 1935.

${ }^{104}$ Interviewé sur les conditions de la chimie universitaire entre les deux guerres, le chimiste Ernest Kahane (ICP, 1924) confirme les problèmes auxquels les universités étaient confrontées. "L'Université était-elle avare? Non, elle était exsangue dans tous les domaines. Les mathématiciens pouvaient peut-être se considérer comme moins pauvres que les autres, car ils avaient moins de besoins, bien qu'ayant comme tout le monde à résoudre le problème épineux des publications, des frais de mission (...) et de chauffage des locaux (...) La situation de la recherche s'est empirée après la Première Guerre mondiale car les crédits étaient minces. C'étaient des crédits "or" jusqu'en 1914 qui sont restés les mêmes après la guerre, quand les dévaluations se sont succédées ». Entretien effectué par Micheline Charpentier et Jean-François Picard, le 12 décembre 1986. Site HISTCNRS, archives orales du CNRS.

105 Voir dans cet ouvrage, l'article de Virginie Fonteneau, «Le cas des thèses d'ingénieur-docteur à Lyon: une nouvelle façon de penser l'enseignement et la recherche en chimie dans l'entre-deux-guerres ».
} 
doivent trouver d'autres solutions. Ainsi, il n'est pas étonnant dans ces conditions que l'Institut chimique de Nancy, premier du genre, créé par Albin Haller, mette en œuvre une réforme aussi ambitieuse que risquée, conduite par son directeur, Alexandre Travers. Il engage une révision complète des programmes de son institution assortie d'un concours d'entrée ouvert aux élèves des classes préparatoires, ce qui diminue de façon conséquente le nombre de candidats et risque de remettre en cause l'existence même de l'école.

«Pour affronter la concurrence, qu'elle soit locale par rapport aux instituts nancéiens ou nationale par rapport aux autres écoles de chimie, l'heure ne semble plus à l'expérimentation, mais au repli sur la valeur sûre que constitue le vivier déjà sélectionné des classes préparatoires $\gg{ }^{106}$.

Faute d'une solution globale, chaque institut a cherché une issue individuelle, accentuant l'hétérogénéité du monde de la formation chimique $^{107}$. Il faudra attendre le décret du 16 janvier 1947 et la mise en œuvre de la réforme visant à transformer les instituts annexes des facultés des sciences en écoles nationales supérieures d'ingénieurs

\footnotetext{
106 Birck Françoise, "Trois écoles d'ingénieurs nancéiennes », in Grelon André et Birck Françoise, Des ingénieurs pour la Lorraine - XIX $-X X^{e}$ siècles, Éditions Serpenoise, Metz, 1998, p.188. F. Birck cite en note les propos de Travers devant les anciens élèves: "La possibilité de l'entrée directe dans nos instituts les fait considérer par beaucoup comme des établissements de deuxième et même de troisième zone! Il est difficile de réagir contre l'opinion, même chez des gens cultivés... » Ibid. note 93 .

${ }^{107}$ Un compte rendu du congrès des ingénieurs de 1937 rapporte un exposé sur la formation des ingénieurs chimistes en vue des carrières industrielles. Dans ce cadre, il est indiqué que la discrimination des candidats par le concours d'entrée aux écoles doit tenir compte de la nécessité absolue de ne laisser passer que les vocations. A ce niveau, il est mentionné l'opposition du syndicat à la réforme Travers de Nancy, à savoir le recrutement au niveau des classes préparatoires. I.C., n¹60, aoûtseptembre 1937.
} 
pour qu'une réponse soit apportée au niveau national ${ }^{108}$. Mais la revendication formulée par le syndicat des ingénieurs chimistes de création d'un diplôme national d'ingénieur des arts chimiques DPLG ne sera jamais exaucée.

\section{Les bouleversements de 1936}

Le bulletin arrête sa parution entre mai 1936 et décembre de la même année ${ }^{109}$. La rédaction prie ses lecteurs de l'excuser mais indique que le secrétariat a été pris par les événements depuis le mois de juin et il a été impossible de trouver le temps matériel de faire paraître la revue. En effet, le syndicat a été saisi par le grand mouvement social du Front populaire et il s'est trouvé entraîné dans des transformations qu'il n'avait jamais imaginées. La publication présente un résumé des étapes de ces transformations.

L'Accord Matignon signé le 7 juin 1936 entre une délégation patronale de la CGPF et une délégation CGT représente une divine surprise pour le syndicat. L'article premier stipule en effet que : «la délégation patronale admet l'établissement immédiat de contrats collectifs de travail ». Jusqu'alors il ne pouvait être fait état que de contrats individuels de travail, ces contrats collectifs seront immédiatement traduits en " conventions collectives », terme qui sera désormais employé. L'article 3 indique notamment que :

\footnotetext{
${ }^{108}$ Cette transformation est loin d'être automatique. Elle est prononcée par décret sur rapport du ministre de l'Éducation nationale après proposition du conseil de l'université intéressée et après avis de la commission permanente des écoles nationales supérieures d'ingénieurs (art. 2).

${ }^{109}$ I.C., n ${ }^{\circ} 156$, décembre 1936.
} 
"L'observation des lois s'imposant à tous les citoyens, les employeurs reconnaissent la liberté d'opinion, ainsi que le droit pour les travailleurs d'adhérer librement et d'appartenir à un syndicat professionnel constitué en vertu du livre III du Code du travail. Les employeurs s'engagent à ne pas prendre en considération le fait d'appartenir ou de ne pas appartenir à un syndicat pour arrêter leurs décisions en ce qui concerne l'embauchage, la conduite ou la répartition du travail, les mesures de discipline ou de congédiement ».

C'est de fait la reconnaissance de l'organisation collective professionnelle et sa capacité à entreprendre au nom de la catégorie représentée des négociations avec la partie patronale, ce que le ministre du Travail, Charles Spinasse, commentera en indiquant que «la nouvelle législation du travail faisait de la profession un groupe fermement organisé, une vraie institution de droit public ${ }^{110}$.

Même si l'accord avait été signé entre patrons et ouvriers, les syndicats d'ingénieurs se disent concernés et veulent pouvoir en bénéficier. Dès le 10 juin, la direction du SICF prend contact avec Gounod, le délégué de l'UIC. Le lendemain, elle participe à une réunion du Syndicat des techniciens de l'industrie chimique, une des branches de l'USTEI (Union des syndicats de techniciens et employés de l'industrie), fédération proche depuis longtemps des thèses de la CGT et qui vient d'y adhérer formellement, réunion à laquelle participent également des non-syndiqués. À l'issue de cette réunion, une commission rassemble les diverses composantes et est chargée d'établir un projet de convention collective.

\footnotetext{
${ }^{110}$ Cité par Kolboom Ingo, La revanche des patrons. Le patronat français face au front populaire, Flammarion, Paris, 1986. Exposé du ministre devant le Conseil national économique, en octobre 1936, cité p. 61.
} 
Le 13 juin, le président Florentin expose au CA les premières démarches. Quelques membres posent la question de l'adhésion du syndicat à la CGT. À l'issue de la discussion, un vote unanime se prononce contre cette adhésion. La collaboration sera maintenue avec le syndicat des techniciens, mais uniquement à propos d'une convention concernant les chimistes et ingénieurs chimistes.

Il y a toutefois un problème à régler d'urgence. Le SICF, comme du reste les autres syndicats de l'USIF et la plupart des syndicats d'ingénieurs, accueille en son sein des patrons d'entreprises de chimie, ce qui en fait un syndicat mixte. N'étant pas exclusivement composé de salariés, il ne peut signer des conventions collectives. Il faut transformer le règlement intérieur qui définit qui peut adhérer au syndicat. Devant l'importance d'une telle décision, le Conseil national doit être saisi de la question et les sections régionales consultées. Il faut cependant faire vite. Une réunion est fixée au 27 juin, le Conseil national sera immédiatement suivi du Conseil d'administration qui prendra la décision. Par la suite, une Assemblée générale sera chargée de voter une modification des statuts. Du reste, dès le 13 juin, le syndicat catholique, l'USIC, a créé le Syndicat des ingénieurs salariés, précisément pour ne plus essuyer de reproches quant à sa représentativité ${ }^{111}$.

Le 27 juin, le président Florentin expose les raisons pour lesquelles le syndicat ne doit plus comprendre que des salariés au sens strict. «Sans doute va-t-il perdre d'excellents et dévoués camarades

\footnotetext{
${ }^{111}$ Nathalie Hugot-Piron indique que « ce sera un véritable succès puisque celui-ci [le SIS] va rapidement recruter 9600 ingénieurs diplômés et autodidactes pour revendiquer l'accès aux instances de représentation et d'arbitrage ». Les "cadres âgés ", op. cit., note 76, p.128.
} 
dont Sordes qui préside encore aujourd'hui la séance est le symbole, mais toute question de sentiment doit être écartée en la circonstance ». Sordes lui-même donne son approbation, et dans le même mouvement, Florentin propose la nomination de l'intéressé comme président d'honneur, ce qui est accepté par acclamation. Après avis conforme du Conseil national, le CA ajoute au chapitre Admission du règlement intérieur le texte suivant :

«Ne peuvent faire partie du syndicat: les ingénieurs qui sont propriétaires d'établissements industriels, commerciaux ou gérants de sociétés en commandite; qui sont membres d'un conseil d'administration d'une entreprise industrielle ou commerciale; qui sont délégués d'un conseil d'administration. Les difficultés qui pourraient surgir pour l'application des règles ci-dessus seront examinées par le conseil d'administration, après avis de la commission d'admission ${ }^{112}$.

Le syndicat pouvait avoir l'illusion qu'une fois ses statuts mis en ordre, la signature de la convention collective suivrait rapidement. Il n'en est rien. La convention de la métallurgie a servi de modèle, mais elle n'a été signée que le 10 juillet $^{113}$, puis, après la période des vacances, ce sont les débats relatifs à la convention des ouvriers de l'industrie chimique qui se sont prolongés. Une première réunion de la

\footnotetext{
${ }^{112}$ I.C., $\mathrm{n}^{\circ} 156$, décembre 1936. Ce numéro rappelle 1'ensemble des décisions qui ont été prises à partir du mois de juin 1936.

113 « L'accord porte sur le droit syndical, la période d'essai, les congés annuels, les allocations familiales, le préavis et les indemnités de congédiements, les brevets d'invention. Elle soumet en outre les rétributions à un barème des appointements, définissant pour la première fois, au-delà du cadre interne des établissements, une rémunération fixée à $1300 \mathrm{f}$. pour les ingénieurs débutants diplômés, et une progression dans l'ancienneté établissant le salaire à $2750 \mathrm{f}$. pour les ingénieurs ayant travaillé huit ans dans l'entreprise ou dix ans dans l'industrie ». Ibid., p. 130.
} 
commission «Collaborateurs» est fixée au 30 septembre ${ }^{114}$. Toutefois, les problèmes ne font que commencer : il faut constituer la commission, puis déterminer quels textes seront examinés et décider s'il y aura une convention spécifique pour les ingénieurs. Mais la délégation des collaborateurs s'y est opposée et pour ne pas rompre l'unité syndicale, les représentants du SICF ont dû adopter cette thèse. Finalement, en date du 9 novembre, il a été acquis qu'il y aurait une convention spéciale pour les ingénieurs.

Un nouveau numéro ne paraît qu'en avril 1937 avec, encore une fois, les excuses de la publication pour cette parution irrégulière $^{115}$. Il est entièrement consacré à l'Assemblée générale du syndicat, le 20 mars 1937. L'orateur, Dubois, revient sur l'épisode de la mise en cuvre des négociations pour l'établissement de la convention collective de la métallurgie de la Région parisienne, qui a servi de matrice aux autres négociations ${ }^{116}$. Les négociations ont duré trois semaines, jour et nuit. Il a fallu se faire admettre car l'USTEI prétendait représenter tous les collaborateurs, des ingénieurs aux employés. Ensuite une collaboration a pu s'établir entre l'USIF, le

\footnotetext{
${ }^{114}$ Le terme « collaborateurs » est usité dans l'entre-deux-guerres pour désigner la couche des employés dépositaires d'une certaine responsabilité et d'une autorité hiérarchique. Il se crée ainsi un Groupement syndical des collaborateurs diplômés des grandes écoles et des facultés (GSCD) en 1937. Le terme cadre qui est également employé dans l'entre-deux-guerres se substitue peu à peu à cette dénomination. Les ingénieurs tiennent à se distinguer de cette catégorie. C'est ainsi qu'on parlera des ingénieurs et cadres. Voir Grelon André, «Les débuts des cadres ", in Bouffartigue Paul (dir.), Cadres : la grande rupture, Éd. La Découverte, Paris, 2001, avec la collaboration d'André Grelon, Guy Groux, Jacqueline Laufer, Yves-Frédéric Livian. Voir p. 21-34.

${ }^{115}$ I.C. $\mathrm{n}^{\circ} 157$, avril 1937.

${ }^{116}$ Il est vraisemblable que Dubois, ingénieur chimiste, mais membre de la direction de l'USIF, participait aux négociations au titre de cette Union et non du seul syndicat des chimistes.
} 
SIS, l'USTEI, la CFTC et la Société amicale des contremaîtres de la métallurgie. La convention n'est certes pas parfaite, mais elle a constitué un précédent d'une importance considérable. Elle se situe comme une suite de la convention collective des ouvriers de la métallurgie de la région parisienne, en vertu d'un engagement de la délégation patronale d'une convention collective pour les catégories de personnel non prévue par la convention des ouvriers.

C'est ce qui est arrivé avec l'UIC qui a d'abord signé une convention avec les ouvriers. Le temps avait passé, les esprits étaient plus calmes et notamment à la CGT, bien différente des délégués de la métallurgie. Une convention spéciale a été décidée pour les ingénieurs, mais la délégation a participé à la convention des collaborateurs pour les employés, techniciens et agents de maîtrise, parce que certains camarades ingénieurs chimistes se trouvaient occupés comme chimistes et même comme aides chimistes. Il ne fallait pas les exclure, d'où un article particulier pour les appointements. Toutefois, le principe est que dans l'industrie chimique, c'est le diplôme qui classe l'ingénieur. La convention de la chimie a été signée le 30 janvier 1937.

Des indications sont données sur des négociations dans d'autres branches et sur d'autres régions. La liste dressée donne le sentiment d'un foisonnement. En réalité, il n'en sera rien. Seules les grandes industries signeront des conventions concernant les ingénieurs, soit au total, 45 conclues jusqu'en 1939, alors qu'entre 
1936 et 1938, près de 5000 conventions seront signées entre patrons et ouvriers $^{117}$.

Mais Dubois livre aussi une information d'une grande importance car ce qui s'annonce est une transformation totale de l'univers syndical des ingénieurs. C'est la création de la Fédération nationale des syndicats d'ingénieurs (FNSI) par la réunion de trois structures syndicales antérieures. L’Union sociale d'ingénieurs catholiques, créée en 1906, avait au départ rassemblé des anciens élèves de l'École centrale avant de s'ouvrir à d'autres ingénieurs. Utilisant au départ la modalité syndicale pour des raisons de commodité de fonctionnement, elle avait progressivement intégré des raisonnements et des revendications de type syndical, notamment après la Première guerre mondiale. C'était le syndicat le plus important en nombre. Il rassemblait à la fois des ingénieurs patrons et des salariés. Il avait intégré une fédération d'associations d'anciens élèves et de syndicats d'ingénieurs, la FASSFI, en 1929, à sa création. En 1936, l'USIC est le premier syndicat à créer un syndicat « bis », le Syndicat des ingénieurs salariés, pour pouvoir participer aux négociations qui s'annoncent sur le plan social. La FASSFI réagit négativement à cette décision, ce qui conduit l'USIC à quitter cette fédération.

Nous avons vu que le SICF avait modifié ses statuts pour ne conserver que les salariés dans le syndicat. La même opération avait été engagée dans les deux autres syndicats de l'Union.

${ }^{117}$ Les « cadres âgés », op. cit., note 76, p. 128. 
La FASSFI (Fédération des associations, sociétés et syndicats français d'ingénieurs) regroupait des associations d'anciens élèves parmi les plus huppées, une société savante, la Société des ingénieurs civils de France, ainsi que l'USIC et un petit syndicat très conservateur, la Chambre syndicale des ingénieurs. Sollicitée pour y adhérer lors de sa création, l'USIF avait refusé, exposant notamment que cette fédération était essentiellement le porte-parole du patronat. Dans un premier temps, la FASSFI s'oppose à la transformation des syndicats mixtes d'ingénieurs en syndicats de salariés. Finalement, pour ne pas être exclue de l'ensemble du mouvement social qui se développe, et après la signature de la convention parisienne de la métallurgie pour les ingénieurs, elle est contrainte de créer son propre syndicat de salariés, le SPID, Syndicat professionnel d'ingénieurs diplômés français, le 31 octobre 1936, dans le but d'organiser un contrefeu à l'alliance qui s'organisait entre le SIS et l'USIF ${ }^{118}$.

Dubois ne dément pas cette alliance, bien au contraire. Il expose que les relations qui se nouaient avec le SIS s'inscrivent dans la suite des rapports noués depuis des années avec l'USIC, syndicat confessionnel qui ne s'en cachait pas. Ce n'était pas un reproche bien qu' " il n'y ait pas de raison qu'il y ait un syndicat catholique et pas un syndicat protestant », petite pique d'un syndicat qui avait toujours hautement revendiqué son caractère laïque.

«Ceci étant dit, il faut constater que l'USIC depuis déjà longtemps, quoique ayant un nombre de patrons assez important, était un groupement qui avait réellement des idées syndicales et qui défendait incontestablement les mêmes thèses

${ }^{118} I d .$, p. 129. 
que nous et les mêmes revendications purement professionnelles des ingénieurs ».

Par conséquent la collaboration avec l'USIC et désormais le SIS apparaît tout à fait naturelle. L’orateur apparaît plus réservé quant au SPID mais les enjeux sont tels qu'il faut passer sur les différends anciens.

«Lorsque le 13 janvier, nous sommes allés voir - je dis nous, il s'agissait d'une délégation composée de l'USIF, du SIS, et du SPID - lorsque nous sommes allés voir M. Lebas, ministre du Travail, à propos de la loi sur l'arbitrage obligatoire, la première chose qu'il nous a dite: "Trois syndicats d'ingénieurs? Pourquoi trois syndicats d'ingénieurs? Pourquoi ne vous fédérez-vous pas ?" ».

En réalité, les contacts, prudents au départ, avaient été pris dès la création du SPID, fin octobre 1936, aboutissant à la création d'un comité de coordination, lequel finit par envisager la création d'une Fédération. Les statuts de la FNSI sont déposés le 20 février 1937. Dotés désormais d'une « maison commune »,

«[les ingénieurs] sont maintenant amenés à discuter entre eux, après s'être affrontés pendant tant d'années. D'une situation de conflits et d'antagonismes, ils sont désormais conduits à discuter sur des actions communes ${ }^{119}$.

La question sociale va être au cœur de leur action, notamment la question des retraites qui, d'une position de recherche d'une protection individuelle, sera désormais socialisée. Le problème des « ingénieurs âgés » qui avait fait tant couler d'encre syndicale sans autre effet qu'une dénonciation devenue un rituel, est désormais traité dans le cadre d'organismes spécialisés de gestion.

${ }^{119}$ Les « cadres âgés », op. cit., note 76, p. 133. 
Plus largement, ce qui apparaît comme un phénomène radicalement nouveau, est la fondation du syndicalisme des cadres avec la constitution de la Confédération générale des cadres de l'économie française (CGCEF) en $1937^{120}$. En août 1944, la CGCEF, la FNSI et le GSCD se regrouperont pour former le Comité national de coordination des syndicats de cadres, ingénieurs et agents de maîtrise qui deviendra le 15 octobre 1944, la Confédération générale des cadres $(\mathrm{CGC})^{121}$.

\section{Conclusion : vers un autre syndicalisme}

Le syndicat des chimistes continue à publier son bulletin jusqu'en 1939. Cependant, désormais les pages sont vides de toutes les grandes campagnes qui avaient été menées, depuis sa fondation, début 1919 jusqu'à juin 1936. Les problèmes qui hantaient de longue date les militants ont été traités de façon absolument inattendue par des moyens qui paraissaient antérieurement inenvisageables et les dirigeants syndicaux ont été conduits à mener des actions communes avec des adversaires devenus des partenaires pour obtenir satisfaction de revendications qui relevaient jusqu'alors du domaine de l'utopie. La loi du 10 juillet 1934 qui avait validé la protection du titre d'ingénieur diplômé et avalisé l'ensemble des instituts chimiques par

\footnotetext{
120 Elle regroupe le Syndicat des ingénieurs de Lille, le Syndicat des cadres aéronautiques, la Fédération nationale des ingénieurs des mines, le Syndicat des cadres de direction des assurances, le Syndicat professionnel des cadres du pétrole. D'autres syndicats rejoindront ensuite la confédération.

121 Mouriaux René, «Le syndicalisme des ingénieurs et cadres. Histoire et historiographies» in Culture technique, 12 (mars 1984), $\mathrm{n}^{\circ}$ spécial «Les ingénieurs », p. 221-227, voir notamment le tableau "Arbre généalogique de la CGC », p. 220.
} 
la commission des titres d'ingénieurs a pris alors une nouvelle dimension. Elle a permis de définir de façon objective la catégorie d'ingénieur diplômé : est ingénieur diplômé celui qui a été formé dans un établissement habilité, dont la liste est publiée dans le Journal Officiel, et qui a vu ses études sanctionnées positivement à l'issue du cursus. Une législation aussi claire est précieuse dans le cadre des négociations des conventions collectives, et parce qu'elle est sans ambiguïté, c'est elle qui permet précisément d'obtenir des conventions spécifiques pour les ingénieurs. Jusqu'alors dans le monde de l'entreprise, le terme ingénieur signifiait une fonction déterminée par l'employeur. Désormais la profession est reconnue et le diplôme s'impose comme un facteur de distinction, notamment vis-à-vis des chimistes sans diplôme, des cadres et autres collaborateurs. Les conventions fixent des barèmes d'entrée dans le monde du travail pour les jeunes ingénieurs et organisent la progression dans la carrière. Dans ces conditions, l'ardente obligation, portée par le syndicat pendant plus d'une décennie, de la création d'un diplôme d'ingénieur chimiste DPLG s'efface, d'autant que le SICF avait pu percevoir à quel point l'administration de l'Éducation nationale, sans s'opposer ouvertement aux propositions syndicales, avait tout mis en œuvre pour étouffer progressivement le projet. Ainsi, la solution spécifique aux chimistes imaginée et défendue par son syndicat était devenue obsolète dans ce nouveau cadre argumentaire.

Il en est de même de la question des «vieux ingénieurs » lancée et portée par l'USIF et reprise et amplifiée dans chacun des trois syndicats qui la composaient, en fonction des caractéristiques de chacun des groupes professionnels représentés. Ce thème, perçu 
comme sensible dans le monde des ingénieurs avait été alors pris en compte, chacun à sa manière, par les autres syndicats d'ingénieurs et de cadres, notamment l'USIC, de façon incantatoire, en mettant l'accent sur la charge émotionnelle que pouvait représenter une telle injustice. À partir de 1936, la prise en charge des fins de carrière a été organisée collectivement, de façon plus rationnelle que la proposition de financement des anciens sans ressource par des dons gracieux et l'ouverture de maisons de vieux chimistes. Certes, toutes les revendications des ingénieurs n'ont pas encore été satisfaites, mais un processus est lancé qui se poursuivra avec la mise en place de l'Agence de retraite complémentaire des cadres, l'AGIRC, en 1947, puis, en 1954, la création de l'Association pour l'emploi des cadres, ingénieurs et techniciens (APEC) ${ }^{122}$.

Même si le SICF avait été membre de l'USIF et, à ce titre, répercutant les mots d'ordre lancés par l'Union, il avait développé une politique de «l'entre-soi», se basant sur la spécificité de la chimie dans le monde industriel, et sur les formes historiques particulières de transmission des savoirs et savoir-faire, opposant cette discipline aux autres filières de formation, ainsi que René Sordes l'avait développé longuement, dans des assemblées syndicales, avant d'en faire un ouvrage. Cette analyse «scientifique » avait fondé l'élaboration d'un axe revendicatif sur l'organisation rationnelle de l'enseignement de la chimie, et partant, sur la valorisation de la carrière du chimiste. À de nombreuses reprises, les textes publiés dans le bulletin dénonçaient la position minorée des professionnels qualifiés de la chimie, les situant

\footnotetext{
122 Voir in: Les "cadres âgés », op. cit., note 76, le chapitre : "La création de l'Association pour l'emploi des cadres, ingénieurs et techniciens (APEC)", p. 209238.
} 
systématiquement au dernier rang de la hiérarchie industrielle, cette vulgate doloriste ayant pour fonction de souder la communauté des chimistes dont le syndicat se désignait le seul protecteur, bataillant pour leur redonner leur « vraie » place.

Parallèlement, le syndicat des ingénieurs électriciens tenait un discours de la même eau, revendiquant, lui aussi, une revalorisation des carrières de ses membres, insuffisamment reconnus. De leur côté, les anciens élèves des Arts et Métiers qui avaient obtenu de haute lutte un brevet (et non un diplôme) d'ingénieur ${ }^{123}$, après des années de réclamation, considéraient que, pour autant, ils se retrouvaient toujours dans les rangs inférieurs de la hiérarchie industrielle, leur progression étant systématiquement bloquée par des ingénieurs de catégorie supérieure. Dans d'autres spécialités industrielles, des exigences similaires étaient émises. Le petit monde de l'ingénierie était ainsi agité de tensions et de querelles, d'autant plus qu'au sein d'un même ensemble de formation disciplinaire, les écoles se faisaient concurrence pour prendre rang dans une hiérarchie de compétence et de prestige, ce qui devait se retrouver aussi bien dans l'organisation de l'entreprise, les diplômés des différentes écoles étant les uns envers les autres des rivaux potentiels, qu'au sein même des syndicats catégoriels : dans ces rassemblements égalitaires, certains, selon la formule célèbre, étaient plus égaux que d'autres. C'est la raison pour laquelle, en période de crise économique, des pressions étaient exercées sur les écoles considérées comme les plus faibles pour qu'elles diminuent le nombre de places offertes au recrutement, voire

\footnotetext{
${ }^{123}$ Les écoles d'arts et métiers relevaient de l'enseignement technique, branche du système éducatif dans laquelle le brevet était l'attestation de la réussite finale des études.
} 
qu'elles ferment boutique... Dans de telles conditions, les revendications, émises par des syndicats dispersés et antagonistes, rassemblant de maigres troupes, avaient peu de chance d'aboutir. Mais le rapport de force devint tout autre lorsque, pris dans le mouvement ouvrier auquel ils n'avaient pas adhéré, dans les grèves auxquelles ils n'avaient pas participé, dans les occupations d'usines qu'ils dénonçaient, les ingénieurs, auprès de qui s’ajoutait désormais la force montante des cadres, eurent l'opportunité de se saisir de l'Accord Matignon qui, a priori ne les concernait pas, pour en tirer avantage et s'inscrire dans les négociations... à condition qu'ils apparaissent comme un collectif et non comme un conglomérat, devant un patronat défait et comme frappé de stupeur ${ }^{124}$. À lui seul, le SICF qui entretenait depuis l'origine des relations ambivalentes avec l'UIC, n'aurait pas été en mesure de négocier une convention pour les ingénieurs, mais la FNSI pouvait représenter l'ensemble de la catégorie professionnelle et imposer, dans cette période conjoncturelle de confusion du monde patronal, un accord profitable. Du reste, le

\footnotetext{
${ }^{124}$ Ingo Kolboom rapporte les propos d'une conférence de Georges Lamirand au Centre des jeunes patrons dans lesquels il décrit «ces groupes mornes que formaient, dans bien des endroits, des collaborateurs complètement débordés par des ouvriers voyant en eux les anciens instruments d'une discipline contre laquelle ils cherchaient à se révolter, et, d'autre part, abandonnés par les patrons qui, trop absorbés, peut-être, par les décisions qu'ils avaient à prendre dans leurs syndicats, avaient oublié même, dans bien des cas, de donner les consignes les plus simples ». Il cite également le témoignage d'un ancien élève du Centre de préparation aux affaires de Paris, exposant que «l'ingénieur était dans sa grande majorité hostile à toute association professionnelle. Il a fallu les événements de juin dernier pour qu'il existât une communauté, de sentiments d'une part et d'intérêts d'autre part, entre tous les ingénieurs. Il découvrit avec une naïveté à peine croyable le syndicalisme et au sein de la plupart des associations d'anciens élèves, ce fut une pluie de lettres, demandes de renseignement... et de reproches ». Kolboom Ingo, « Le patronat et les cadres (1936-1938) », in Bouvier Jean (dir.), La France en mouvement, 1934-1938, Éditions Champ Vallon, Paris, 1986, p. 237.
} 
patronat lui-même, ou tout au moins dans ses branches les plus éclairées, ne se montra pas hostile à la création de la CGCEF, en 1937, y voyant un contrepoids utile à la puissante $\mathrm{CGT}^{125}$.

Ce syndicalisme cadre était encore hésitant et en devenir en cette fin de III $^{\mathrm{e}}$ République. Il faudra attendre la Libération pour voir s'amorcer un puissant mouvement qui s'affirme autonome, tant vis-àvis du syndicalisme ouvrier que de la représentation patronale ${ }^{126}$. Le syndicalisme autonome, particulier à chaque industrie, tel celui de l'industrie chimique, aura alors vécu. C'est désormais au sein d'une fédération de branche industrielle affiliée à une Confédération que les chimistes pourront agir syndicalement.

\footnotetext{
${ }^{125}$ Kolboom, Ibid., p. 249-256.

${ }^{126}$ La Confédération générale des cadres doit combattre pour être habilitée. Le 25 mars 1946, elle lance sa première grève générale. Suivra une série de meetings et de manifestations pour qu'enfin elle soit reconnue par décision des pouvoirs publics du 8 avril 1948 comme une organisation avec une "présomption irréfragable » de représentativité habilitée à la négociation collective pour ce qui concerne les cadres. Quatre mois plus tard, la loi du 23 août 1948 adapte la législation de la sécurité sociale à la situation des cadres.
} 


\section{Annexe 1}

\section{Recrutement : liste établie en 1919}

\section{Catégories de chimistes accédant au syndicat}

(selon le règlement intérieur) :

"Il s'agit de différencier ceux qui ont été formés dans des institutions spécialisés de ceux qui, à des titres divers sont arrivés plus tardivement à la chimie. Pour cela, la mesure est la durée d'activité dans l'industrie ${ }^{127}$.

1. Anciens élèves diplômés d'écoles préparant spécialement aux carrières chimiques: 25 ans d'âge et un ou deux ans de carrière industrielle ${ }^{128}$

a. École centrale des arts et manufactures, section chimie

b. Chimie Bordeaux

c. Chimie industrielle Lyon

d. Chimie Mulhouse

e. Physique chimie industrielle de Paris, section chimie

f. Institut de chimie appliquée Paris

g. Institut chimique Lille

h. Institut chimique Nancy

i. Institut chimique Montpellier

j. Institut chimique Toulouse

2. Anciens élèves des écoles officielles préparant aux carrières industrielles et anciens élèves des facultés des sciences, avec 3 années de pratique

a. École centrale des arts et manufactures

b. Écoles des mines de Paris et de Saint-Etienne

c. École Polytechnique

d. Institut national agronomique

e. Institut industriel du Nord

\footnotetext{
${ }^{127}$ I.C., n 1 , décembre 1919.

${ }^{128}$ L'École de chimie de Rouen, organisée par la Société industrielle rouennaise, créée en 1917, ne figure pas dans la liste de même que celle de Marseille ouverte la même année par la Chambre de commerce de la ville. L'École de chimie de Strasbourg, créée en 1919 au sein de la nouvelle université française de cette ville est encore en pleine installation, de même que l'Institut de chimie industrielle (ICI) de la Faculté catholique des sciences de Lyon. En revanche la chaire de chimie de Besançon qui délivre un diplôme d'ingénieur depuis le 17 février 1914, l'Institut de chimie de Clermont-Ferrand, créée par la Chambre de commerce du Puy-de-Dôme en 1911 et associé à l'Université de Clermont-Ferrand depuis 1913, l'Institut de chimie de l’Université de Caen ouvert en 1914 ne sont pas mentionnés.
} 
f. Facultés des sciences, docteurs ou licenciés avec deux certificats de chimie dont CS de chimie générale

g. Pharmaciens diplômés

h. EPCI (section physique)

i. Élèves de la première catégorie ayant accompli toute leur scolarité

3. Anciens élèves d'écoles pratiques ou spéciales devenus chimistes : six années de pratique dont trois à direction technique ou responsabilité effective

a. École des sciences de Rouen

b. École centrale lyonnaise

c. Écoles d'agriculture ou établissements similaires qui pourraient être créés

d. Laboratoires du Muséum ou du CNAM

4. Chimistes n'appartenant à aucune des autres catégories : 10 ans de pratique dont 3 de direction technique ou responsabilité effective

\section{Sections professionnelles}

1. Grosse industrie chimique, acides, engrais phosphatés et azotés, soudières, sels de potasse, poudres et explosifs, électrochimie

2. Produits chimiques, purs, pharmaceutiques, photographiques

3. Matières colorantes et teintures, parfums et produits plastiques

4. Caoutchouc, corps gras, couleurs et vernis

5. Combustibles, métallurgie, électrométallurgie, céramique

6. Produits agricoles, industrie des fermentations, cuirs, peaux, colles

7. Enseignement technique, labos de recherche, chimistesconseils, experts 


\title{
Annexe 2
}

\section{Projet de loi de diplôme d'ingénieur chimiste DPLG}

\author{
Projet présenté devant l'assemblée générale du syndicat, \\ le 25 janvier $1925^{129}$
}

\section{PROJET DE LOI}

Art.1. - il est créé un diplôme d'Ingénieur-Chimiste DPLG délivré dans les conditions prévues aux articles suivants et signé du ministre de l'Instruction publique.

Art.2. - Un jury sera chaque année chargé pour la France et ses colonies, de faire subir aux candidats des épreuves dont chacune sera éliminatoire, épreuves écrites, pratiques et orales.

Art.3. - Le programme d'examen sera éliminatoire, épreuves écrites, pratiques et orales.

Art.3 [sic] - Le programme d'examen sera fixé par une Commission comprenant les représentants :

1. De l'Enseignement supérieur

2. Des Administrations de l'État

3. Des Industriels

4. Des diplômés

proposés par les intéressés et choisis par le ministre.

Art. 4. - Le jury d'examen sera choisi par le ministre, sur proposition de la commission prévue à l'article précédent.

Art. 5. - Un règlement d'administration publique mis en vigueur six mois après la promulgation de la présente loi déterminera les règlements concernant le jury, la commission et les examens prévus par la présente loi. Art. 6. - Les possesseurs de diplômes d'Ingénieurs-Chimistes ou de titres équivalents, délivrés antérieurement à la promulgation de la présente loi, pourront obtenir à titre individuel le diplôme d'Ingénieur-Chimiste DPLG sur demande adressée au ministre de l'Instruction publique. Ces demandes accompagnées de l'exposé des travaux et référence des requérants, seront soumis obligatoirement pour avis à la commission prévue à l'article 3 de la présente Loi ou d'une sous-commission désignée par elle.

${ }^{129}$ I.C., n³3, mars 1925. 
Le projet est complété lors du congrès de 1928 du syndicat par l'adoption de la proposition d'appellation du nouveau diplôme : "Diplôme d’Ingénieur des Arts Chimiques » par 1768 voix contre 64.

Le vœu suivant est adopté à l'unanimité :

Le Congrès du Syndicat des ingénieurs chimistes français, réuni le 11 février 1928, dans la salle du Musée social, à l'unanimité des 1832 délégués présents ou représentés,

Donne mandat au Conseil d'administration du Syndicat des ingénieurs chimistes français de poursuivre plus énergiquement encore que par le passé, la création d'un diplôme d'État d'Ingénieur des Arts Chimiques en accord avec l'Union des industries chimiques et l'Union nationale des associations d'anciens élèves des écoles de chimie et l'Union nationale des étudiants ;

La première étape de l'action devant être la nomination, au ministère de l'Instruction publique (Direction de l'Enseignement supérieur), d'une commission où figurerait: des représentants de l'Enseignement supérieur, de l'Enseignement technique; des industriels désignés par l'Union des industries chimiques; des diplômés désignés par l'Union nationale des associations d'anciens élèves des écoles de chimie ; de l'Union nationale des étudiants ${ }^{130}$.

${ }^{130}$ I.C., n ${ }^{\circ} 66$, mars 1928. 